\title{
1. PRIMARY MINERALOGY OF LEG 118 GABBROIC ROCKS AND THEIR PLACE IN THE SPECTRUM OF OCEANIC MAFIC IGNEOUS ROCKS ${ }^{1}$
}

\author{
R. Hébert, ${ }^{2}$ M. Constantin, ${ }^{2}$ and P. T. Robinson ${ }^{3}$
}

\begin{abstract}
We present results of a microprobe investigation of fresh and least-deformed and metamorphosed gabbroic rocks from Leg 118, Hole 735B, drilled on the east side of the Atlantis II Fracture Zone, Southwest Indian Ridge. This rock collection comprises cumulates ranging from troctolites to olivine-gabbro and olivine-gabbronorite to ilmenite-rich ferrogabbros and ferrogabbronorites. As expected, the mineral chemistry is variable and considerably expands the usual oceanic reference spectrum. Olivine, plagioclase, and clinopyroxene are present in all the studied samples. Orthopyroxene and ilmenite, although not rare, are not ubiquitous. Olivine compositions range from $\mathrm{Fo}_{85}$ to $\mathrm{Fo}_{30}$, while plagioclase compositions vary from $\mathrm{An}_{79}$ to $\mathrm{An}_{27} \cdot \mathrm{Mg} /\left(\mathrm{Mg}+\mathrm{Fe}^{2+}\right.$ ) of clinopyroxene (mostly diopside to augite) varies from 0.88 to $0.54 . \mathrm{Mg} /\left(\mathrm{Mg}+\mathrm{Fe}^{2+}\right)$ of orthopyroxene varies from 0.84 to 0.50 . These minerals are not significantly zoned. All mineralogical data indicate that fractional crystallization is an important factor for the formation of cumulates. However, sharp contacts, interpreted as layering boundaries or intrusion margins, suggest polycyclic fractionation of several magma batches of limited volumes. Calculated compositions of magmas in equilibrium with the most magnesian mineral samples at the bottom of the hole represent fractionated liquids through separation of olivine, plagioclase, and clinopyroxene at moderate to low pressures (less than $9 \mathrm{~kb}$ ). Crystallization of orthopyroxene and ilmenite occurs in the most differentiated liquids. Mixing of magmas having various compositions before entering the cumulate zone is another mechanism necessary to explain extremely differentiated iron-rich gabbros formed in this slow-spreading ridge environment.
\end{abstract}

\section{INTRODUCTION}

About $0.5 \mathrm{~km}$ of gabbroic rocks, ranging in composition from troctolites to ferrogabbros, was drilled during Leg 118 on the eastern margin of the Atlantis II Fracture Zone of the Southwest Indian Ridge system. The high recovery $(87 \%)$ provides an excellent section of part of oceanic layer 3 that was created at a slow-spreading ridge. This stratigraphically controlled suite of gabbroic rocks is the most complete one ever recovered from the ocean floor. Here, we report the primary mineralogy of selected samples and attempt to understand the magmatic processes that generated the observed textures and mineralogies. The Atlantis II gabbros are compared with other gabbros recovered at an ultrafast-spreading ridge (the Garrett Fracture Zone) and at a slow-spreading ridge (the Mid-Cayman Rise). Implications for magma genesis at constructive plate boundaries are discussed in terms of primitive magma compositions, crystal fractionation, and magma mixing.

\section{ANALYTICAL PROCEDURES}

From a total of 204 polished thin sections (eight shipboard thin sections and 96 post-cruise thin sections), 143 were selected for microprobe analysis. The analyzed samples represent the different types of gabbro occurring at different depths in Hole 735B (Fig. 1), and are thought to be representative of each of the major lithologic units defined by the shipboard party (Robinson, Von Herzen, et al., 1989). In addition, selected samples displaying modal or grain-size layering were analyzed. Two to four analyses were performed

\footnotetext{
${ }^{1}$ Von Herzen, R. P., Robinson, P. T., et al., 1991. Proc. ODP, Sci. Results, 118: College Station, TX (Ocean Drilling Program).

2 Département de Géologie, Université Laval, Ste-Foy, Québec, Canada GIK 7P4.

${ }^{3}$ Centre For Marine Geology, Dalhousie University, Halifax, Nova Scotia, Canada B3H $3 \mathrm{~J} 5$.
}

on each grain to detect zoning or heterogeneity. Pyroxenes containing thin (less than $5 \mu \mathrm{m}$ ) exsolution lamellae were analyzed using a defocused beam that covered about $16 \mu \mathrm{m}^{2}$. This procedure results in apparently homogeneous compositions near the pre-exsolution compositions of the high-temperature pyroxenes. Large exsolution lamellae $(>5 \mu \mathrm{m})$ were analyzed both individually, with a focused beam, and globally, using the previously described procedure. Major, minor, and trace elements ( $\mathrm{Ti}, \mathrm{Al}, \mathrm{Ni}, \mathrm{Cr}$ ) were analyzed using an accelerating voltage of $15 \mathrm{kV}$ and counting times of $100 \mathrm{~s}$. Representative mineral analyses are given in Tables 1 through 6. Amphiboles are considered to be largely secondary and are not included in this study. Covariation diagrams were constructed using averages of homogeneous phases.

\section{MEGASCOPIC FEATURES}

The Leg 118 Shipboard Party divided the recovered sections into six lithologic units (hereafter termed Units I through VI; Robinson, Von Herzen, et al., 1989). Units I and II consist of olivine-bearing to olivine-poor gabbros and gabbronorites that have been variably affected by high-temperature plastic deformation and mylonitization. This process dramatically reduced the initial grain size of the foliated gabbros and largely obliterated the primary structures and textures. Deformation is much less intense in Units III to VI, and both sharp and gradational contacts involving grain-size, modal, and phase contrasts are commonly preserved. Variations in grain size are by far the most frequent (e.g., Samples 118-735B-38R-3, 58-64 cm; 118-735B-44R-2, 115-122 cm; and 118-735B-79R-7, $104-109 \mathrm{~cm})$, and they are thought to be of magmatic origin because phase compositions vary across the boundaries. Such contacts were probably formed either by phase layering or intrusion. Some of these contacts are difficult to interpret because they may have been plastically transposed in response to underlying asthenospheric flowage of the type that affects the lower cumulates in many ophiolite complexes (Nicolas et al., 1988). Such deformation may also be partly 


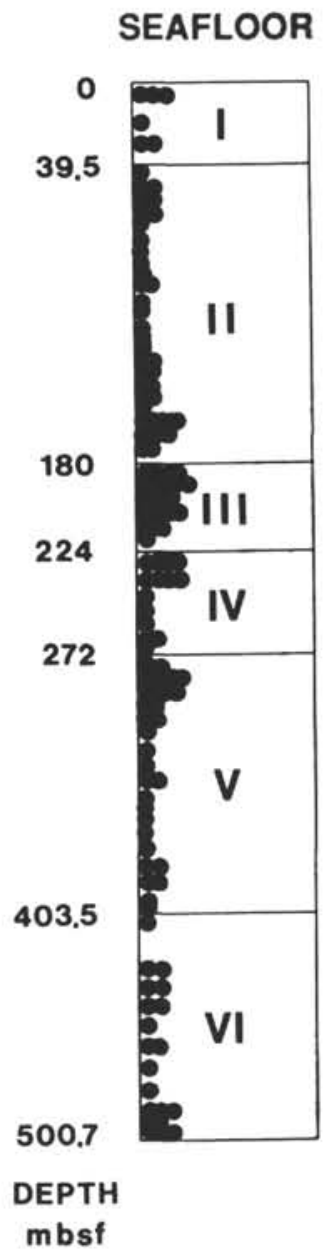

Foliated Metagabbros:
porphyroclastic, mylonitic and gabbronorites

\author{
Layered Gabbros: \\ mesocumulate olivine-rich to olivine - \\ poor gabbros, some Fe-Ti-oxide- \\ bearing gabbros. Some mylonitic and \\ porphyroclastic gabbros.
}

\author{
Layered Gabbros: \\ laminated mesocumulate olivine- \\ to Fe-Ti-oxide-bearing gabbros. \\ Some mylonitic zones.
}

\section{Layered and Foliated Gabbros: granular Fe-Ti-oxide-evolved gabbros intruded by trondjhemite.}

\section{Layered Gabbros \\ mesocumulate olivine-gabbro breccias \\ cemented by epidote, albite, chlorite, \\ diopside.}

\section{Layered Gabbros: orthocumulate to granular olivine- and troctolitic gabbros some mylonitic to porphyroclastic zones.}

Figure 1. Hole summary with lithologic units (according to Robinson, Von Herzen, et al., 1989) and location of microprobed thin sections. Numbers are meters below seafloor for unit boundaries and bottom of Hole 735B.

responsible for the mylonitic and porphyroclastic zones encountered in the drilled section. Some of the deformation may also be related to listric normal faulting at the ridge crest during crustal accretion.

\section{MICROSCOPIC FEATURES}

Most of the studied samples exhibit cumulus textures that range from adcumulus to mesocumulus. Chromite, olivine, plagioclase, clinopyroxene, orthopyroxene, and ilmenite are the common cumulus phases, and textural relationships indicate the presence of little trapped melt. Pyroxenes commonly ophitically enclose other phases, particularly plagioclase. Plagioclase occurs in large, subhedral grains that typically show preferred orientations. Olivine is typically anhedral and commonly corroded. Large oikocrysts of clinopyroxene and orthopyroxene (up to $11 \mathrm{~cm}$ long), which contain smaller crystals of olivine and plagioclase, are oriented perpendicular to the observed layering. Most ilmenite grains are anhedral, reflecting extensive deformation. In the deformed rocks, ilmenite typically fills intergranular spaces between recrystallized silicate phases. Exsolution lamellae are common in both clinopyroxene (including pigeonite) and orthopyroxene. The presence of narrow exsolution lamellae can cause analytical problems and lead to apparent variations in $\mathrm{CaO}$ and $\mathrm{FeO}$ (see
"Analytical Procedures" section, this chapter). Broad exsolution bands and wormlike blebs ( $>5 \mu \mathrm{m}$ wide) commonly cut across the mineral cleavage. Such exsolution features and the presence of pigeonite are typical of slowly cooled plutonic rocks (Hodges and Papike, 1976; Robinson, 1980). Similar textural relationships have been described from xenoliths enclosed in ferrobasalt from the Juan de Fuca Ridge (Hekinian et al., 1985; Eaby et al., 1986). However, these xenoliths have at least partially re-equilibrated with the host magma.

\section{WHOLE-ROCK GEOCHEMISTRY}

To provide a better framework for our mineralogical studies, we have used the shipboard geochemical data (Robinson, Von Herzen, et al., 1989) to develop a downhole stratigraphy for Hole 735B (Fig. 2). Whole-rock geochemical logs show oscillatory variations for $\mathrm{Mg} /\left(\mathrm{Mg}+\mathrm{Fe}^{2+}\right)$, normative Fo and $\mathrm{An}$, as well as for trace elements $\mathrm{Ni}, \mathrm{Cr}$, and $\mathrm{TiO}_{2}$. There commonly is a correlation between lithologic boundaries and geochemical breaks. The boundary between Units V and VI is defined by a downward increase in modal olivine and is not marked by a geochemical break (Robinson, Von Herzen, et al., 1989). The upper parts of the sequence (Units I and II) have been plastically deformed, but igneous textures are preserved in the other lithologic units. In addition, many of 
the fine-grained metamorphic rocks have been hydrothermally altered.

We attribute the apparent cyclic geochemical variations in the rocks of Units I and II to reflect the combined effects of original lithological variation, metamorphism, and deformation, with associated hydrothermal alteration. Lithological variation in the sequence is also indicated by immobile trace elements, such as $\mathrm{Y}$ and $\mathrm{Zr}$ (Robinson, Von Herzen et al., 1989). Cyclic variations were also observed in Units III and IV. Below Unit IV, only $\mathrm{Ni}, \mathrm{Cr}$, and normative An exhibit cyclical variations. $\mathrm{Ni}$ and $\mathrm{Cr}$ are compatible elements that correlate with modal abundances of olivine and chromite plus pyroxene, respectively. Cyclical variations in these elements reflect magmatic layering and cryptic variations in mineral compositions.

There is a systematic inverse correlation between $\mathrm{Ti}$ and $\mathrm{Ni}, \mathrm{Cr}, \mathrm{Mg} /\left(\mathrm{Mg}+\mathrm{Fe}^{2+}\right)$, normative $\mathrm{Fo}$, and normative $\mathrm{An}$. $\mathrm{TiO}_{2}$ values correlate with the modal abundance of ilmenite that is present at various stratigraphic levels. Maximum $\mathrm{TiO}_{2}$ values (9 wt\%) occur in Unit IV, but other peaks are present in Units I and II. The available geochemical data suggest that ilmenite is concentrated in the most evolved cumulates found in Hole 735B. For instance, ilmenite-rich layers have both Fe-rich olivine $\left(\mathrm{Fo}_{30}\right)$ and Na-rich plagioclase $\left(\mathrm{An}_{30}\right)$. The ilmenite is probably a magmatic phase produced by fractionation of an iron-rich melt. This interpretation is supported by the $\mathrm{Mg}$ and $\mathrm{Mn}$ contents of ilmenite (Table 5).

Units V and VI are more homogeneous than the overlying rocks and show little variation in $\mathrm{Mg} /\left(\mathrm{Mg}+\mathrm{Fe}^{2+}\right.$ ) (average value 0.85), $\mathrm{TiO}_{2}$ (average 0.10 wt\%) and normative Fo (average value 80 ). $\mathrm{Ni}$ and $\mathrm{Cr}$ are abundant throughout this part of the sequence and attain values as high as 741 and 3668 ppm, respectively. These rocks have high, but somewhat variable, normative $A n$ contents $\left(\mathrm{An}_{60-80}\right)$. The geochemical data suggest that the cumulates of Units V and VI were derived from a relatively primitive, high-magnesian melt.

Thus, the rocks of Hole 735B range from Fe-rich to $\mathrm{Mg}$-rich cumulates, with all gradations in between. These end-member compositions, as well as the intermediate types, occur at various stratigraphic levels, but the Fe-rich cumulates are concentrated in Unit IV and the $\mathrm{Mg}$-rich varieties are concentrated in Units V and VI.

$\mathrm{Zr} / \mathrm{Y}$ ratios vary from 0.8 to 4 , but exhibit no systematic variation with depth. The relatively wide range of $\mathrm{Zr} / \mathrm{Y}$ ratios may reflect different parental magmas or different abundances of clinopyroxene in the cumulates (KDY-CPX $=0.5$ to 1.5 , Pearce and Norry, 1979). If different magma types were involved, intrusive relationships must be invoked to explain the lack of stratigraphic control on the $\mathrm{Zr} / \mathrm{Y}$ ratios.

\section{NORMATIVE MINERAL COMPOSITIONS VS. MICROPROBE COMPOSITIONS}

In Figure 2, we have plotted actual olivine and plagioclase compositions determined by microprobe analysis vs. CIPW normative mineral compositions calculated by bulk rock compositions. In general, the two show good agreement, but some discrepancies were observed for An values in Unit IV and below $380 \mathrm{~m}$. These discrepancies probably reflect abrupt geochemical and mineralogical variations with depth associated with phase layering. Normative compositions are usually good indicators of the expected mineralogy in cumulate gabbros. However, normative values represent averages of short core intervals that mask real variations in mineral composition across the same intervals. Differences are most pronounced in magnesian gabbros and ferrogabbros, where normative calculations do not reflect the actual distribution of $\mathrm{Mg}$ in olivine and pyroxene and of $\mathrm{Fe}$ in oxides and other silicate minerals.
The differences in measured and calculated $\mathrm{Mg} /\left(\mathrm{Mg}+\mathrm{Fe}^{2+}\right)$, as shown in Figure 2, clearly illustrate this relationship. Even though the trends in normative and measured $\mathrm{Mg} /\left(\mathrm{Mg}+\mathrm{Fe}^{2+}\right)$ are parallel, the normative mineral compositions obviously do not reflect small-scale mineralogical variations related to crystal accumulation and subsolidus re-equilibration. As will be seen later, these variations also do not reflect mineral zoning. In Fe-rich gabbros, the differences between $\mathrm{Mg} /(\mathrm{Mg}$ $\left.+\mathrm{Fe}^{2+}\right)$ of clinopyroxene and normative $\mathrm{Mg} /\left(\mathrm{Mg}+\mathrm{Fe}^{2+}\right)$ is easily explained by exsolution of orthopyroxene lamellae, which increases the $\mathrm{Mg} /\left(\mathrm{Mg}+\mathrm{Fe}^{2+}\right)$ of the host grain.

\section{ZONING OF MINERALS}

Figure 3 shows the range of compositions measured in single grains of olivine, plagioclase, and clinopyroxene. Clearly, compositional zoning is not significant in these minerals over a wide range of composition. Plagioclase and clinopyroxene are somewhat more variable in composition than olivine, but measured values represent less than $10 \%$ molecular variation relative to total mineral composition. This variation lies within the range of normal mineral heterogeneity from cation site occupancy. The absence of significant zoning is in agreement with low abundances of trapped liquid, deduced from textural relationships and low incompatible element abundances (Meyer et al., 1989). The small volume of trapped liquid probably reflects the mode of mineral accumulation or later expulsion of intercumulus liquids. The absence of such liquids in these rocks probably explains the lack of important post-cumulus re-equilibration, such as was reported by Chalokwu and Grant (1987) for the Duluth layered intrusion.

\section{MINERAL CHEMISTRY}

Analyzed compositions for olivine, plagioclase, and clinopyroxene are shown in Figure 4. Olivine $\left(\mathrm{Fo}_{84-58}\right)$ coexists with clinopyroxene of calcic augitic composition $(\mathrm{Mg} /(\mathrm{Mg}+$ $\left.\mathrm{Fe}^{2+}\right)=0.85-0.65$ ) and plagioclase ranging from $\mathrm{An}_{79}$ to $\mathrm{An}_{50}$. Evolved oxide-bearing gabbros are characterized by iron-rich olivine $\left(\mathrm{Fo}_{35-30}\right)$, augitic clinopyroxene $\left(\mathrm{Mg} /\left[\mathrm{Mg}+\mathrm{Fe}^{2+}\right]=\right.$ $0.55)$ and sodic plagioclase $\left(\mathrm{An}_{31-27}\right)$. The evolved gabbros contain feldspar, which is more Na-rich, and olivine, which is more Fe-rich, than is usually reported for oceanic gabbros (see Elthon, 1987, for a review). These new findings involve a considerable extension of the oceanic compositional spectrum. These mineral assemblages reflect Fenner-type differentiation of tholeiitic magmas. Significant enrichment in Fe and $\mathrm{Ti}$ leads to crystallization of ilmenite, such as that observed in Unit IV. Large variations in clinopyroxene composition are largely attributed to subsolidus re-equilibration. Application of the Lindsley and Anderson (1983) geothermometer (not illustrated here) suggests a temperature range of $1200^{\circ}$ to $700^{\circ} \mathrm{C}$ for crystallization and, thus, important subsolidus re-equilibration of these rocks.

\section{TRACE ELEMENTS IN SILICATE MINERALS}

Variations in trace elements $(\mathrm{Cr}, \mathrm{Ni}$, and $\mathrm{Ti})$ in mineral phases were used to test various fractionation models derived from variations in major elements and to identify different magma batches. In Figure $5 \mathrm{~A}$, variation of $\mathrm{NiO}$ content in olivine is plotted vs. forsterite content. Maximum $\mathrm{NiO}$ values of $0.18 \mathrm{wt} \%$ were recorded in the most mafic rocks, those of Units V and VI, although Fe-rich olivine from Section 118735B-80R-6 has no detectable $\mathrm{NiO}$. In general, the lower limit of detection for $\mathrm{NiO}$ in olivine is at $\mathrm{Fo}_{65}$. The observed variation in $\mathrm{NiO}$ is consistent with rapid removal of $\mathrm{Ni}$ from the melt by incorporation in olivine (KD $=10-15$; Irving, 1978) and sulfide phases (small amounts of pentlandite and 
Table 1. Representative microprobe analyses of olivine from Leg 118 plutonic rocks.

\begin{tabular}{|c|c|c|c|c|c|c|c|c|c|c|c|}
\hline $\begin{array}{c}\text { Core/section: } \\
\text { Interval }(\mathrm{cm}) \text { : } \\
\text { Piece: } \\
\text { Unit: }\end{array}$ & $\begin{array}{c}8 \mathrm{D}-1 \\
29-32 \\
5 \\
\text { I }\end{array}$ & $\begin{array}{c}8 \mathrm{D}-1 \\
29-32 \\
5 \\
\mathrm{I}\end{array}$ & $\begin{array}{c}13 \mathrm{R}-1 \\
102-105 \\
11 \mathrm{~B} \\
\text { II }\end{array}$ & $\begin{array}{c}40 \mathrm{R}-3 \\
137-145 \\
6 \\
\text { III }\end{array}$ & $\begin{array}{c}42 \mathrm{R}-4 \\
115-123 \\
5 \mathrm{~A} \\
\text { III }\end{array}$ & $\begin{array}{c}42 \mathrm{R}-4 \\
115-123 \\
5 \mathrm{~A} \\
\text { III }\end{array}$ & $\begin{array}{c}44 \mathrm{R}-3 \\
37-40 \\
5 \mathrm{D} \\
\text { III }\end{array}$ & $\begin{array}{c}44 \mathrm{R}-3 \\
37-40 \\
5 \mathrm{D} \\
\text { III }\end{array}$ & $\begin{array}{c}45 \mathrm{R}-2 \\
19-24 \\
1 \\
\text { III }\end{array}$ & $\begin{array}{c}51 \mathrm{R}-3 \\
110-114 \\
\text { IE } \\
\text { IV }\end{array}$ & $\begin{array}{c}51 \mathrm{R}-3 \\
110-114 \\
1 \mathrm{E} \\
\text { IV }\end{array}$ \\
\hline $\mathrm{SiO}_{2}$ & 37.63 & 37.54 & 37.93 & 34.99 & 35.07 & 35.14 & 38.13 & 38.05 & 38.42 & 33.85 & 33.70 \\
\hline $\mathrm{TiO}_{2}$ & 0.00 & 0.00 & 0.00 & 0.00 & 0.00 & 0.00 & 0.00 & 0.00 & 0.00 & 0.00 & 0.00 \\
\hline $\mathrm{Al}_{2} \mathrm{O}_{3}$ & 0.00 & 0.00 & 0.00 & 0.00 & 0.00 & 0.00 & 0.00 & 0.00 & 0.00 & 0.00 & 0.00 \\
\hline $\mathrm{Cr}_{2} \mathrm{O}_{3}$ & 0.00 & 0.00 & 0.00 & 0.00 & 0.00 & 0.00 & 0.00 & 0.00 & 0.00 & 0.00 & 0.00 \\
\hline $\mathrm{FeO}$ & 22.23 & 22.70 & 25.67 & 35.13 & 34.05 & 33.87 & 19.48 & 19.51 & 17.42 & 44.49 & 46.58 \\
\hline $\mathrm{MnO}$ & 0.63 & 0.61 & 0.52 & 0.59 & 0.81 & 0.86 & 0.37 & 0.38 & 0.43 & 0.71 & 0.74 \\
\hline $\mathrm{NiO}$ & 0.00 & 0.00 & 0.00 & 0.00 & 0.00 & 0.00 & 0.00 & 0.09 & 0.09 & 0.00 & 0.00 \\
\hline $\mathrm{MgO}$ & 38.99 & 39.16 & 36.54 & 29.00 & 29.98 & 30.07 & 42.11 & 41.84 & 44.00 & 21.38 & 19.30 \\
\hline $\mathrm{CaO}$ & 0.06 & 0.07 & 0.03 & 0.00 & 0.00 & 0.00 & 0.04 & 0.00 & 0.00 & 0.00 & 0.00 \\
\hline $\mathrm{Na}_{2} \mathrm{O}$ & 0.00 & 0.00 & 0.00 & 0.00 & 0.00 & 0.00 & 0.00 & 0.00 & 0.00 & 0.00 & 0.00 \\
\hline $\mathrm{K}_{2} \mathrm{O}$ & 0.00 & 0.00 & 0.00 & 0.00 & 0.00 & 0.00 & 0.00 & 0.00 & 0.00 & 0.00 & 0.00 \\
\hline Total: & 99.54 & 100.08 & 100.69 & 99.71 & 99.91 & 99.94 & 100.13 & 99.87 & 100.36 & 100.43 & 100.32 \\
\hline $\mathrm{Si}$ & 0.982 & 0.975 & 0.995 & 0.971 & 0.966 & 0.967 & 0.973 & 0.975 & 0.968 & 0.981 & 0.991 \\
\hline $\mathrm{Ti}$ & 0.000 & 0.000 & 0.000 & 0.000 & 0.000 & 0.000 & 0.000 & 0.000 & 0.000 & 0.000 & 0.000 \\
\hline Al & 0.000 & 0.000 & 0.000 & 0.000 & 0.000 & 0.000 & 0.000 & 0.000 & 0.000 & 0.000 & 0.000 \\
\hline $\mathrm{Cr}$ & 0.000 & 0.000 & 0.000 & 0.000 & 0.000 & 0.000 & 0.000 & 0.000 & 0.000 & 0.000 & 0.000 \\
\hline $\mathrm{Fe}$ & 0.485 & 0.493 & 0.563 & 0.815 & 0.784 & 0.779 & 0.416 & 0.418 & 0.367 & 1.078 & 1.145 \\
\hline Mn & 0.014 & 0.013 & 0.012 & 0.014 & 0.019 & 0.020 & 0.008 & 0.008 & 0.009 & 0.017 & 0.018 \\
\hline $\mathrm{Ni}$ & 0.000 & 0.000 & 0.000 & 0.000 & 0.000 & 0.000 & 0.000 & 0.002 & 0.002 & 0.000 & 0.000 \\
\hline $\mathrm{Mg}$ & 1.517 & 1.516 & 1.429 & 1.200 & 1.231 & 1.234 & 1.602 & 1.598 & 1.653 & 0.924 & 0.846 \\
\hline $\mathrm{Ca}$ & 0.002 & 0.002 & 0.001 & 0.000 & 0.000 & 0.000 & 0.001 & 0.000 & 0.000 & 0.000 & 0.000 \\
\hline $\mathrm{Na}$ & 0.000 & 0.000 & 0.000 & 0.000 & 0.000 & 0.000 & 0.000 & 0.000 & 0.000 & 0.000 & 0.000 \\
\hline K & 0.000 & 0.000 & 0.000 & 0.000 & 0.000 & 0.000 & 0.000 & 0.000 & 0.000 & 0.000 & 0.000 \\
\hline Total & 3.000 & 3.000 & 3.000 & 3.000 & 3.000 & 3.000 & 3.000 & 3.000 & 3.000 & 3.000 & 3.000 \\
\hline Fo & 75.2 & 75.0 & 71.3 & 59.1 & 60.5 & 60.7 & 79.1 & 78.9 & 81.5 & 45.7 & 42.1 \\
\hline $\mathrm{Fa}$ & 24.8 & 25.0 & 28.7 & 40.9 & 39.5 & 39.3 & 20.9 & 21.1 & 18.5 & 54.3 & 57.9 \\
\hline
\end{tabular}

Ni-pyrrhotite were observed). The nearly continuous evolution from $\mathrm{Mg}$-olivine to $\mathrm{Fe}$-olivine suggests that only limited resorption of the newly formed crystals took place. Gabbros from the Mid-Cayman Rise and the Garrett Fracture Zone are characterized by Ni-rich olivine that crystallized from more primitive (or S-undersaturated) melts than those from which the Leg 118 gabbros were formed (Sato, 1977).

Figure $5 \mathrm{~B}$ is a plot of $\mathrm{Cr}_{2} \mathrm{O}_{3}$ contents vs. $\mathrm{Mg} /\left(\mathrm{Mg}+\mathrm{Fe}^{2+}\right)$ in clinopyroxene. Maximum $\mathrm{Cr}_{2} \mathrm{O}_{3}$ values (up to $1.0 \mathrm{wt} \%$ ) are recorded in Unit VI $\left(\mathrm{Mg} /\left[\mathrm{Mg}+\mathrm{Fe}^{2+}\right]=0.85\right) . \mathrm{Cr}_{2} \mathrm{O}_{3}$ contents decrease rapidly in the more evolved samples $(0.5 \mathrm{wt} \%$ for $\left.\mathrm{Mg} /\left[\mathrm{Mg}+\mathrm{Fe}^{2+}\right]=0.80\right)$. The detection limit of $\mathrm{Cr}_{2} \mathrm{O}_{3}$ in clinopyroxene is reached at $\mathrm{Mg} /\left(\mathrm{Mg}+\mathrm{Fe}^{2+}\right)=0.72$. Again, such variation is compatible with fractionation of clinopyroxene from a Cr-bearing, magnesian melt. $\mathrm{Cr}_{2} \mathrm{O}_{3}$ is also partitioned into chromite and orthopyroxene (not shown here). Clinopyroxenes from the Garrett Fracture Zone are enriched in $\mathrm{Cr}_{2} \mathrm{O}_{3}$ relative to those from Hole $735 \mathrm{~B}$, mainly because orthopyroxene is not abundant in such $\mathrm{Mg}$ gabbros (Hébert et al., 1983), leaving more $\mathrm{Cr}$ to be partitioned into clinopyroxene. Gabbroic rocks of the Mid-Cayman Rise have clinopyroxenes that exhibit a chemical evolution trend similar to those from the Leg 118 gabbros. $\mathrm{Cr}_{2} \mathrm{O}_{3}$ variation in clinopyroxene is consistent with experimental results for moderate to low pressures of fractionation (Grove and Bryan, 1983).

Variations in $\mathrm{TiO}_{2}$ content of clinopyroxene vs. Fs molecular proportions are shown in Figure 5C. Maximum $\mathrm{TiO}_{2}$ values (1.5 wt\%) were observed in Fs-poor clinopyroxenes from Units V and VI. Minimum values $(0.1 \mathrm{wt} \%)$ were recorded in Unit $\mathrm{I}$. However, $\mathrm{TiO}_{2}$ contents of clinopyroxene are highly variable, even within a single unit. Clinopyroxenes from oxide-bearing layers in Units V and VI are Fs-rich varieties having moderate $\mathrm{TiO}_{2}$ contents $(0.3-0.6$ wt\%). $\mathrm{TiO}_{2}$ decreases in clinopyroxenes as the Fs component increases. Clearly, $\mathrm{TiO}_{2}$ has been preferentially parti- tioned into ilmenite, which appears on the liquidus of highly fractionated melts.

Variations in trace elements strongly suggest that the Leg 118 gabbros were formed from more than one batch of magma. $\mathrm{NiO}$ and $\mathrm{Cr}_{2} \mathrm{O}_{3}$ gradually decrease upward in the section between Units VI and IV. Values increase abruptly at the base of Unit III and then decrease gradually upward through Units III and II. A complete data set is not available for Unit $\mathrm{I}$. $\mathrm{TiO}_{2}$ varies reversely to $\mathrm{NiO}$ and $\mathrm{Cr}_{2} \mathrm{O}_{3}$. Magma mixing provides the best explanation for these trends. In Figure 5C, a mixing line shows how fractionation trend I might have been shifted to trend II. This mechanism should involve at least two different magma batches and might have been repeated several times. Mixing of residual liquid left after crystallization of the early formed gabbros (Units VI, V, and part of IV) with a primitive liquid might have reset the compatible trace elements to the higher values observed in gabbros of Units IV through I. The parallel increase of the Fs molecule in clinopyroxene is also consistent with "normal" differentiation of a tholeiitic melt. Replenishment of the magma reservoir may have caused Fs to decrease in response to an increase in $\mathrm{Mg}$ (Unit III, Fig. 5C). Replenishment of the magma reservoir with a more primitive melt may also have caused ilmenite growth to cease, so that $\mathrm{TiO}_{2}$ should be partitioned into clinopyroxene $\left(\mathrm{TiO}_{2}\right.$ content increases in clinopyroxene, see Fig. 5C). This general scheme of fractionation and mixing is well documented in major ophiolitic complexes, such as Semail (Pallister and Hopson, 1981) and the Bay of Islands (Komor et al., 1985, 1987), and we think that this scheme was important during the genesis of Leg 118 gabbros. The origin of the Fe-rich layers in Units $\mathrm{V}$ and VI remains problematic. These layers (e.g., Section 118-735B-80R-6) might be explained by local replenishment of the magma reservoir or by intrusion of small batches of evolved (and already mixed) liquid.

Additional information about the magmatic evolution of these gabbros may be obtained by considering the $\mathrm{Ti}$ vs. $\mathrm{Cr}$ 
Table 1 (continued).

\begin{tabular}{|c|c|c|c|c|c|c|c|c|c|c|c|c|c|c|c|c|c|c|c|c|c|c|}
\hline $\begin{array}{c}54 \mathrm{R}-4 \\
0-9 \\
1 \\
\text { IV }\end{array}$ & $\begin{array}{c}54 \mathrm{R}-4 \\
0-9 \\
1 \\
\text { IV }\end{array}$ & $\begin{array}{c}54 \mathrm{R}-4 \\
0-9 \\
1 \\
\text { IV }\end{array}$ & $\begin{array}{c}54 \mathrm{R}-4 \\
0-9 \\
1 \\
\text { IV }\end{array}$ & $\begin{array}{c}61 \mathrm{R}-1 \\
126-135 \\
5 \\
\mathrm{~V}\end{array}$ & $\begin{array}{c}69 \mathrm{R}-2 \\
55-67 \\
2 \mathrm{~B} \\
\mathrm{~V}\end{array}$ & $\begin{array}{c}74 \mathrm{R}-5 \\
84-92 \\
3 \mathrm{~B} \\
\mathrm{~V}\end{array}$ & $\begin{array}{c}74 \mathrm{R}-6 \\
0-8 \\
1 \\
\mathrm{~V}\end{array}$ & $\begin{array}{c}75 \mathrm{R}-5 \\
27-30 \\
1 \mathrm{~A} \\
\mathrm{~V}\end{array}$ & $\begin{array}{c}79 \mathrm{R}-6 \\
105-111 \\
4 \\
\text { VI }\end{array}$ & $\begin{array}{c}79 \mathrm{R}-7 \\
104-109 \\
9 \\
\text { VI }\end{array}$ & $\begin{array}{c}79 \mathrm{R}-7 \\
104-109 \\
9 \\
\text { VI }\end{array}$ & $\begin{array}{c}80 \mathrm{R}-1 \\
117-123 \\
12 \\
\text { VI }\end{array}$ & $\begin{array}{c}80 \mathrm{R}-6 \\
121-129 \\
14 \\
\text { VI }\end{array}$ & $\begin{array}{c}81 R-1 \\
130-132 \\
1 \\
\text { VI }\end{array}$ & $\begin{array}{c}81 \mathrm{R}-6 \\
77-86 \\
4 \mathrm{~B} \\
\mathrm{VI}\end{array}$ & $\begin{array}{c}82 \mathrm{R}-4 \\
12-20 \\
1 \mathrm{~B} \\
\mathrm{VI}\end{array}$ & $\begin{array}{c}83 \mathrm{R}-6 \\
53-59 \\
3 \\
\text { VI }\end{array}$ & $\begin{array}{c}84 \mathrm{R}-7 \\
13-23 \\
1 \mathrm{~A} \\
\mathrm{VI}\end{array}$ & $\begin{array}{c}85 \mathrm{R}-7 \\
0-5 \\
1 \mathrm{~A} \\
\text { VI }\end{array}$ & $\begin{array}{c}86 \mathrm{R}-4 \\
64-70 \\
3 \mathrm{~B} \\
\text { VI }\end{array}$ & $\begin{array}{c}86 \mathrm{R}-6 \\
28-38 \\
4 \\
\text { VI }\end{array}$ & $\begin{array}{c}87 \mathrm{R}-6 \\
106-114 \\
14 \\
\text { VI }\end{array}$ \\
\hline 32.80 & 32.64 & 32.65 & 36.98 & 38.69 & 37.64 & 39.02 & 36.86 & 37.27 & 37.69 & 38.12 & 37.79 & 39.30 & 32.63 & 38.46 & 38.84 & 38.44 & 39.51 & 37.29 & 39.17 & 37.29 & 38.27 & 38.74 \\
\hline 0.00 & 0.00 & 0.00 & 0.00 & 0.00 & 0.00 & 0.00 & 0.00 & 0.00 & 0.00 & 0.00 & 0.00 & 0.00 & 0.00 & 0.00 & 0.00 & 0.00 & 0.00 & 0.00 & 0.00 & 0.00 & 0.00 & 0.00 \\
\hline 0.00 & 0.00 & 0.00 & 0.00 & 0.00 & 0.00 & 0.00 & 0.00 & 0.00 & 0.00 & 0.00 & 0.00 & 0.00 & 0.00 & 0.00 & 0.00 & 0.00 & 0.00 & 0.00 & 0.00 & 0.00 & 0.00 & 0.00 \\
\hline 0.00 & 0.00 & 0.00 & 0.00 & 0.00 & 0.00 & 0.00 & 0.00 & 0.00 & 0.00 & 0.00 & 0.00 & 0.00 & 0.00 & 0.00 & 0.00 & 0.00 & 0.00 & 0.00 & 0.00 & 0.00 & 0.00 & 0.00 \\
\hline 51.47 & 51.56 & 52.35 & 26.34 & 21.73 & 22.19 & 22.93 & 31.27 & 20.38 & 17.82 & 24.26 & 26.21 & 16.77 & 50.08 & 22.78 & 18.89 & 18.42 & 15.56 & 26.24 & 17.40 & 28.42 & 22.05 & 18.31 \\
\hline 1.02 & 1.04 & 1.47 & 0.59 & 0.40 & 0.42 & 0.43 & 0.63 & 0.49 & 0.38 & 0.48 & 0.50 & 0.34 & 1.16 & 0.50 & 0.45 & 0.38 & 0.31 & 0.53 & 0.30 & 0.73 & 0.52 & 0.33 \\
\hline 0.00 & 0.00 & 0.04 & 0.00 & 0.06 & 0.16 & 0.03 & 0.00 & 0.00 & 0.05 & 0.03 & 0.03 & 0.09 & 0.00 & 0.03 & 0.04 & 0.03 & 0.03 & 0.02 & 0.06 & 0.01 & 0.08 & 0.06 \\
\hline 14.41 & 14.13 & 13.17 & 36.18 & 38.84 & 39.61 & 38.27 & 32.11 & 40.97 & 42.26 & 37.68 & 36.65 & 43.89 & 15.74 & 38.83 & 41.08 & 43.08 & 44.68 & 36.17 & 43.17 & 34.15 & 39.57 & 42.62 \\
\hline 0.10 & 0.10 & 0.08 & 0.00 & 0.08 & 0.07 & 0.03 & 0.07 & 0.00 & 0.03 & 0.00 & 0.00 & 0.00 & 0.12 & 0.07 & 0.05 & 0.06 & 0.05 & 0.13 & 0.06 & 0.02 & 0.03 & \\
\hline 0.00 & 0.00 & 0.00 & 0.00 & 0.00 & 0.00 & 0.00 & 0.00 & 0.00 & 0.00 & 0.00 & 0.00 & 0.00 & 0.00 & 0.00 & 0.00 & 0.00 & 0.00 & 0.00 & 0.00 & 0.00 & 0.00 & 0.00 \\
\hline 0.00 & 0.00 & 0.00 & 0.00 & 0.00 & 0.00 & 0.00 & 0.00 & 0.00 & 0.00 & 0.00 & 0.00 & 0.00 & 0.00 & 0.00 & 0.00 & 0.00 & 0.00 & 0.00 & 0.00 & 0.00 & 0.00 & 0.00 \\
\hline 99.80 & 99.47 & 99.76 & 100.09 & 99.80 & 100.09 & 100.71 & 100.94 & 99.11 & 98.23 & 100.57 & 101.18 & 100.39 & 99.73 & 100.67 & 99.35 & 100.41 & 100.14 & 100.38 & 100.16 & 100.62 & 100.52 & 100.12 \\
\hline 1.002 & 0.999 & 1.005 & 0.978 & 1.008 & 0.975 & 1.011 & 0.990 & 0.966 & 0.975 & 0.993 & 0.987 & 0.990 & 0.987 & 0.996 & 1.002 & 0.975 & 0.993 & 0.984 & 0.993 & 0.993 & 0.987 & 0.984 \\
\hline 0.000 & 0.000 & 0.000 & 0.000 & 0.000 & 0.000 & 0.000 & 0.000 & 0.000 & 0.000 & 0.000 & 0.000 & 0.000 & 0.000 & 0.000 & 0.000 & 0.000 & 0.000 & 0.000 & 0.000 & 0.000 & 0.000 & 0.000 \\
\hline 0.000 & 0.000 & 0.000 & 0.000 & 0.000 & 0.000 & 0.000 & 0.000 & 0.000 & 0.000 & 0.000 & 0.000 & 0.000 & 0.000 & 0.000 & 0.000 & 0.000 & 0.000 & 0.000 & 0.000 & 0.000 & 0.000 & 0.000 \\
\hline 0.000 & 0.000 & 0.000 & 0.000 & 0.000 & 0.000 & 0.000 & 0.000 & 0.000 & 0.000 & 0.000 & 0.000 & 0.000 & 0.000 & 0.000 & 0.000 & 0.000 & 0.000 & 0.000 & 0.000 & 0.000 & 0.000 & 0.000 \\
\hline 1.314 & 1.323 & 1.347 & 0.582 & 0.471 & 0.480 & 0.498 & 0.705 & 0.441 & 0.384 & 0.531 & 0.573 & 0.354 & 1.269 & 0.492 & 0.408 & 0.390 & 0.327 & 0.579 & 0.369 & 0.633 & 0.477 & 0.390 \\
\hline 0.027 & 0.027 & 0.039 & 0.012 & 0.009 & 0.009 & 0.009 & 0.015 & 0.012 & 0.009 & 0.012 & 0.012 & 0.009 & 0.030 & 0.012 & 0.009 & 0.009 & 0.006 & 0.012 & 0.006 & 0.015 & 0.012 & 0.009 \\
\hline 0.000 & 0.000 & 0.003 & 0.000 & 0.003 & 0.003 & 0.000 & 0.000 & 0.000 & 0.003 & 0.000 & 0.000 & 0.003 & 0.000 & 0.000 & 0.003 & 0.000 & 0.000 & 0.000 & 0.003 & 0.000 & 0.003 & 0.003 \\
\hline 0.657 & 0.645 & 0.606 & 1.428 & 1.509 & 1.530 & 1.482 & 1.290 & 1.581 & 1.629 & 1.467 & 1.428 & 1.647 & 0.711 & 1.497 & 1.578 & 1.626 & 1.674 & 1.422 & 1.629 & 1.356 & 1.521 & 1.614 \\
\hline 0.003 & 0.003 & 0.003 & 0.000 & 0.003 & 0.003 & 0.003 & 0.003 & 0.000 & 0.003 & 0.000 & 0.000 & 0.000 & 0.003 & 0.003 & 0.003 & 0.003 & 0.003 & 0.003 & 0.003 & 0.000 & 0.003 & 0.003 \\
\hline 0.000 & 0.000 & 0.000 & 0.000 & 0.000 & 0.000 & 0.000 & 0.000 & 0.000 & 0.000 & 0.000 & 0.000 & 0.000 & 0.000 & 0.000 & 0.000 & 0.000 & 0.000 & 0.000 & 0.000 & 0.000 & 0.000 & 0.000 \\
\hline 0.000 & 0.000 & 0.000 & 0.000 & 0.000 & 0.000 & 0.000 & 0.000 & 0.000 & 0.000 & 0.000 & 0.000 & 0.000 & 0.000 & 0.000 & 0.000 & 0.000 & 0.000 & 0.000 & 0.000 & 0.000 & 0.000 & 0.000 \\
\hline 3.003 & 2.997 & 3.003 & 3.000 & 3.003 & 3.000 & 3.003 & 3.003 & 3.000 & 3.003 & 3.003 & 3.000 & 3.003 & 3.000 & 3.000 & 3.003 & 3.003 & 3.003 & 3.000 & 3.003 & 2.997 & 3.003 & 3.003 \\
\hline 32.9 & 32.3 & 30.4 & 70.6 & 75.9 & 75.8 & 74.5 & 64.2 & 77.7 & 80.6 & 73.0 & 70.9 & 81.9 & 35.4 & 74.8 & 79.1 & 80.3 & 83.4 & 70.6 & 81.3 & 67.7 & 75.7 & 80.2 \\
\hline 67.1 & 67.7 & 69.6 & 29.4 & 24.1 & 24.2 & 25.5 & 35.8 & 22.3 & 19.4 & 27.0 & 29.1 & 18.1 & 64.6 & 25.2 & 20.9 & 19.7 & 16.6 & 29.4 & 18.7 & 32.3 & 24.3 & 19.8 \\
\hline
\end{tabular}


Table 2. Representative microprobe analyses of plagioclase from Leg 118 plutonic rocks.

\begin{tabular}{|c|c|c|c|c|c|c|c|c|c|c|c|c|c|c|c|c|c|c|c|c|c|c|}
\hline $\begin{array}{c}\text { Core/section: } \\
\text { Interval }(\mathrm{cm}) \text { : } \\
\text { Piece: } \\
\text { Unit: }\end{array}$ & $\begin{array}{c}1 D-1 \\
90-92 \\
14 \\
\text { I }\end{array}$ & $\begin{array}{c}\text { 1D-1 } \\
90-92 \\
14 \\
\text { I }\end{array}$ & $\begin{array}{c}8 \mathrm{D}-1 \\
29-32 \\
5 \\
1\end{array}$ & $\begin{array}{c}8 \mathrm{D}-1 \\
29-32 \\
5 \\
\mathrm{I}\end{array}$ & $\begin{array}{c}13 \mathrm{R}-1 \\
102-105 \\
11 \mathrm{~B} \\
\text { II }\end{array}$ & $\begin{array}{c}14 \mathrm{R}-1 \\
26-29 \\
2 \mathrm{~A} \\
\text { II }\end{array}$ & $\begin{array}{c}14 \mathrm{R}-1 \\
26-29 \\
2 \mathrm{~A} \\
\text { II }\end{array}$ & $\begin{array}{c}18 \mathrm{R}-3 \\
37-40 \\
4 \\
\text { II }\end{array}$ & $\begin{array}{c}23 R-3 \\
34-37 \\
2 \\
\text { II }\end{array}$ & $\begin{array}{c}38 \mathrm{R}-3 \\
58-64 \\
2 \\
\text { III }\end{array}$ & $\begin{array}{c}40 \mathrm{R}-3 \\
137-145 \\
6 \\
\text { III }\end{array}$ & $\begin{array}{c}40 \mathrm{R}-3 \\
137-145 \\
6 \\
\text { III }\end{array}$ & $\begin{array}{c}42 \mathrm{R}-4 \\
115-123 \\
5 \mathrm{~A} \\
\text { III }\end{array}$ & $\begin{array}{c}44 \mathrm{R}-3 \\
37-40 \\
5 \mathrm{D} \\
\text { III }\end{array}$ & $\begin{array}{c}44 \mathrm{R}-3 \\
37-40 \\
5 \mathrm{D} \\
\text { III }\end{array}$ & $\begin{array}{c}47 \mathrm{R}-2 \\
5-10 \\
1 \\
\text { IV }\end{array}$ & $\begin{array}{c}47 \mathrm{R}-2 \\
5-10 \\
1 \\
\text { IV }\end{array}$ & $\begin{array}{c}52 \mathrm{R}-4 \\
12-25 \\
1 \\
\text { IV }\end{array}$ & $\begin{array}{c}52 \mathrm{R}-4 \\
12-25 \\
1 \\
\text { IV }\end{array}$ & $\begin{array}{c}54 \mathrm{R}-4 \\
0-9 \\
1 \\
\text { IV }\end{array}$ & $\begin{array}{c}54 \mathrm{R}-4 \\
0-9 \\
1 \\
\text { IV }\end{array}$ & $\begin{array}{c}54 \mathrm{R}-4 \\
0-9 \\
1 \\
\text { IV }\end{array}$ \\
\hline $\begin{array}{l}\mathrm{SiO}_{2} \\
\mathrm{TiO}_{2} \\
\mathrm{Al}_{2} \mathrm{O}_{3} \\
\mathrm{FeO} \\
\mathrm{MnO} \\
\mathrm{MgO} \\
\mathrm{CaO} \\
\mathrm{Na}_{2} \mathrm{O} \\
\mathrm{K}_{2} \mathrm{O}\end{array}$ & $\begin{array}{r}56.96 \\
0.00 \\
27.05 \\
0.09 \\
0.00 \\
0.04 \\
9.19 \\
6.40 \\
0.03 \\
\end{array}$ & $\begin{array}{r}56.02 \\
0.00 \\
27.78 \\
0.15 \\
0.00 \\
0.04 \\
9.83 \\
6.02 \\
0.07 \\
\end{array}$ & $\begin{array}{r}56.14 \\
0.00 \\
27.88 \\
0.05 \\
0.00 \\
0.04 \\
9.76 \\
5.66 \\
0.01 \\
\end{array}$ & $\begin{array}{r}48.47 \\
0.00 \\
33.11 \\
0.11 \\
0.00 \\
0.06 \\
16.17 \\
2.25 \\
0.03 \\
\end{array}$ & $\begin{array}{r}53.79 \\
0.00 \\
29.01 \\
0.13 \\
0.00 \\
0.06 \\
11.79 \\
4.58 \\
0.09 \\
\end{array}$ & $\begin{array}{r}54.10 \\
0.00 \\
29.63 \\
0.10 \\
0.00 \\
0.03 \\
11.57 \\
5.16 \\
0.02 \\
\end{array}$ & $\begin{array}{r}54.09 \\
0.00 \\
29.70 \\
0.21 \\
0.00 \\
0.03 \\
11.75 \\
4.95 \\
0.04 \\
\end{array}$ & $\begin{array}{r}52.91 \\
0.00 \\
29.87 \\
0.08 \\
0.00 \\
0.06 \\
12.49 \\
4.43 \\
0.01 \\
\end{array}$ & $\begin{array}{r}57.99 \\
0.00 \\
25.99 \\
0.15 \\
0.00 \\
0.06 \\
7.92 \\
6.61 \\
0.07 \\
\end{array}$ & $\begin{array}{r}58.53 \\
0.00 \\
26.82 \\
0.25 \\
0.00 \\
0.02 \\
8.29 \\
6.47 \\
0.13 \\
\end{array}$ & $\begin{array}{r}56.37 \\
0.00 \\
27.83 \\
0.17 \\
0.00 \\
0.03 \\
9.26 \\
6.43 \\
0.06 \\
\end{array}$ & $\begin{array}{r}56.91 \\
0.00 \\
27.87 \\
0.07 \\
0.00 \\
0.03 \\
9.25 \\
6.26 \\
0.08 \\
\end{array}$ & $\begin{array}{r}55.68 \\
0.00 \\
28.03 \\
0.32 \\
0.00 \\
0.30 \\
9.76 \\
6.67 \\
0.06 \\
\end{array}$ & $\begin{array}{r}51.69 \\
0.00 \\
30.45 \\
0.18 \\
0.00 \\
0.04 \\
13.56 \\
3.76 \\
0.00 \\
\end{array}$ & $\begin{array}{r}51.34 \\
0.00 \\
30.73 \\
0.16 \\
0.00 \\
0.07 \\
13.73 \\
3.48 \\
0.00 \\
\end{array}$ & $\begin{array}{r}58.35 \\
0.00 \\
26.38 \\
0.18 \\
0.00 \\
0.01 \\
7.93 \\
7.66 \\
0.10 \\
\end{array}$ & $\begin{array}{r}58.15 \\
0.00 \\
26.43 \\
0.16 \\
0.00 \\
0.01 \\
7.91 \\
7.40 \\
0.13 \\
\end{array}$ & $\begin{array}{r}58.88 \\
0.00 \\
25.45 \\
0.32 \\
0.00 \\
0.06 \\
7.51 \\
7.86 \\
0.03 \\
\end{array}$ & $\begin{array}{r}59.35 \\
0.00 \\
26.20 \\
0.10 \\
0.00 \\
0.06 \\
7.76 \\
7.39 \\
0.10 \\
\end{array}$ & $\begin{array}{r}60.22 \\
0.00 \\
25.89 \\
0.15 \\
0.00 \\
0.06 \\
7.12 \\
7.33 \\
0.13 \\
\end{array}$ & $\begin{array}{r}59.93 \\
0.00 \\
25.92 \\
0.32 \\
0.00 \\
0.04 \\
7.40 \\
7.49 \\
0.13 \\
\end{array}$ & $\begin{array}{r}60.50 \\
0.00 \\
25.32 \\
0.15 \\
0.00 \\
0.04 \\
6.45 \\
8.56 \\
0.15 \\
\end{array}$ \\
\hline Total: & 99.76 & 99.91 & 99.54 & 100.20 & 99.45 & 100.61 & 100.77 & 99.85 & 98.79 & 100.51 & 100.15 & 100.47 & 100.82 & 99.68 & 99.51 & 100.61 & 100.19 & 100.11 & 100.96 & 100.90 & 101.23 & 101.17 \\
\hline $\begin{array}{l}\mathrm{Si} \\
\mathrm{Ti} \\
\mathrm{Al} \\
\mathrm{Fe} \\
\mathrm{Mn} \\
\mathrm{Mg} \\
\mathrm{Ca} \\
\mathrm{Na} \\
\mathrm{K}\end{array}$ & $\begin{array}{l}0.560 \\
0.000 \\
1.433 \\
0.003 \\
0.000 \\
0.003 \\
0.442 \\
0.558 \\
0.002 \\
\end{array}$ & $\begin{array}{l}2.518 \\
0.000 \\
1.472 \\
0.006 \\
0.000 \\
0.003 \\
0.473 \\
0.525 \\
0.004\end{array}$ & $\begin{array}{l}2.539 \\
0.000 \\
1.486 \\
0.002 \\
0.000 \\
0.003 \\
0.473 \\
0.496 \\
0.001\end{array}$ & $\begin{array}{l}2.215 \\
0.000 \\
1.783 \\
0.004 \\
0.000 \\
0.004 \\
0.792 \\
0.199 \\
0.002 \\
\end{array}$ & $\begin{array}{l}2.449 \\
0.000 \\
1.557 \\
0.005 \\
0.000 \\
0.004 \\
0.575 \\
0.404 \\
0.005 \\
\end{array}$ & $\begin{array}{l}2.424 \\
0.000 \\
1.565 \\
0.004 \\
0.000 \\
0.002 \\
0.556 \\
0.448 \\
0.001\end{array}$ & $\begin{array}{l}2.424 \\
0.000 \\
1.569 \\
0.008 \\
0.000 \\
0.002 \\
0.564 \\
0.430 \\
0.002\end{array}$ & $\begin{array}{l}2.400 \\
0.000 \\
1.595 \\
0.005 \\
0.000 \\
0.005 \\
0.610 \\
0.390 \\
0.000\end{array}$ & $\begin{array}{l}2.630 \\
0.000 \\
1.390 \\
0.005 \\
0.000 \\
0.005 \\
0.385 \\
0.580 \\
0.005 \\
\end{array}$ & $\begin{array}{l}2.614 \\
0.000 \\
1.412 \\
0.009 \\
0.000 \\
0.001 \\
0.397 \\
0.560 \\
0.007\end{array}$ & $\begin{array}{l}2.521 \\
0.000 \\
1.467 \\
0.006 \\
0.000 \\
0.002 \\
0.444 \\
0.557 \\
0.003\end{array}$ & $\begin{array}{l}2.540 \\
0.000 \\
1.466 \\
0.003 \\
0.000 \\
0.002 \\
0.442 \\
0.542 \\
0.005\end{array}$ & $\begin{array}{l}2.466 \\
0.000 \\
1.463 \\
0.012 \\
0.000 \\
0.020 \\
0.463 \\
0.573 \\
0.003 \\
\end{array}$ & $\begin{array}{l}2.358 \\
0.000 \\
1.637 \\
0.007 \\
0.000 \\
0.003 \\
0.663 \\
0.333 \\
0.000 \\
\end{array}$ & $\begin{array}{l}2.350 \\
0.000 \\
1.658 \\
0.006 \\
0.000 \\
0.005 \\
0.673 \\
0.309 \\
0.000\end{array}$ & $\begin{array}{l}2.580 \\
0.000 \\
1.375 \\
0.007 \\
0.000 \\
0.001 \\
0.376 \\
0.657 \\
0.006\end{array}$ & $\begin{array}{l}2.586 \\
0.000 \\
1.385 \\
0.006 \\
0.000 \\
0.001 \\
0.377 \\
0.638 \\
0.007\end{array}$ & $\begin{array}{l}2.615 \\
0.000 \\
1.330 \\
0.010 \\
0.000 \\
0.005 \\
0.360 \\
0.680 \\
0.005\end{array}$ & $\begin{array}{l}2.635 \\
0.000 \\
1.365 \\
0.005 \\
0.000 \\
0.005 \\
0.365 \\
0.630 \\
0.005 \\
\end{array}$ & $\begin{array}{l}2.665 \\
0.000 \\
1.350 \\
0.005 \\
0.000 \\
0.005 \\
0.340 \\
0.630 \\
0.010 \\
\end{array}$ & $\begin{array}{l}2.640 \\
0.000 \\
1.345 \\
0.010 \\
0.000 \\
0.005 \\
0.350 \\
0.640 \\
0.010 \\
\end{array}$ & $\begin{array}{l}2.650 \\
0.000 \\
1.305 \\
0.005 \\
0.000 \\
0.005 \\
0.300 \\
0.725 \\
0.010 \\
\end{array}$ \\
\hline Total: & 5.000 & 5.000 & 5.000 & 5.000 & 5.000 & 5.000 & 5.000 & 5.000 & 5.000 & 5.000 & 5.000 & 5.000 & 5.000 & 5.000 & 5.000 & 5.000 & 5.000 & 5.005 & 5.000 & 5.005 & 5.000 & 5.000 \\
\hline $\begin{array}{l}\text { An } \\
\mathrm{Ab} \\
\mathrm{Or}\end{array}$ & $\begin{array}{r}44.2 \\
55.7 \\
0.2\end{array}$ & $\begin{array}{r}47.2 \\
52.4 \\
0.4\end{array}$ & $\begin{array}{r}48.8 \\
51.2 \\
0.1\end{array}$ & $\begin{array}{r}79.7 \\
20.1 \\
0.2\end{array}$ & $\begin{array}{r}58.4 \\
41.1 \\
0.5\end{array}$ & $\begin{array}{r}55.3 \\
44.6 \\
0.1\end{array}$ & $\begin{array}{r}56.6 \\
43.2 \\
0.2\end{array}$ & $\begin{array}{r}61.0 \\
39.0 \\
0.0\end{array}$ & $\begin{array}{r}39.7 \\
59.8 \\
0.5\end{array}$ & $\begin{array}{r}41.1 \\
58.1 \\
0.8\end{array}$ & $\begin{array}{r}44.2 \\
55.5 \\
0.3\end{array}$ & $\begin{array}{r}44.7 \\
54.8 \\
0.5\end{array}$ & $\begin{array}{r}44.6 \\
55.1 \\
0.3\end{array}$ & $\begin{array}{r}66.6 \\
33.4 \\
0.0\end{array}$ & $\begin{array}{r}68.6 \\
31.4 \\
0.0\end{array}$ & $\begin{array}{r}36.2 \\
63.3 \\
0.5\end{array}$ & $\begin{array}{r}36.9 \\
62.4 \\
0.7\end{array}$ & $\begin{array}{r}34.4 \\
65.1 \\
0.5\end{array}$ & $\begin{array}{r}36.5 \\
63.0 \\
0.5\end{array}$ & $\begin{array}{r}34.7 \\
64.3 \\
1.0\end{array}$ & $\begin{array}{r}35.0 \\
64.0 \\
1.0\end{array}$ & $\begin{array}{r}29.0 \\
70.0 \\
1.0\end{array}$ \\
\hline
\end{tabular}


Table 2 (continued).

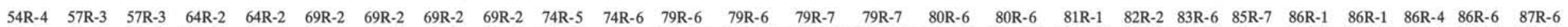

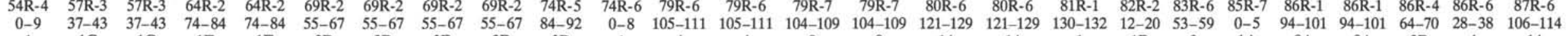

\begin{tabular}{|c|c|c|c|c|c|c|c|c|c|c|c|c|c|c|c|c|c|c|c|c|c|c|c|c|c|}
\hline $\begin{array}{c}1 \\
\text { IV }\end{array}$ & $\begin{array}{l}1 \mathrm{C} \\
\mathrm{V}\end{array}$ & $\begin{array}{l}1 \mathrm{C} \\
\mathrm{V}\end{array}$ & $\begin{array}{c}1 \mathrm{E} \\
\mathrm{V}\end{array}$ & $\begin{array}{c}1 \mathrm{E} \\
\mathrm{V}\end{array}$ & $\stackrel{2 \mathrm{~B}}{\mathrm{~V}}$ & $\underset{\mathrm{V}}{2 \mathrm{~B}}$ & $\stackrel{2 \mathrm{~B}}{\mathrm{~V}}$ & $\underset{\mathrm{V}}{2 \mathrm{~B}}$ & $\begin{array}{l}3 \mathrm{~B} \\
\mathrm{~V}\end{array}$ & $\begin{array}{l}1 \\
\mathrm{~V}\end{array}$ & $\begin{array}{c}4 \\
\text { VI }\end{array}$ & $\begin{array}{c}4 \\
\mathrm{VI}\end{array}$ & $\begin{array}{c}9 \\
\text { VI }\end{array}$ & $\begin{array}{c}9 \\
\text { VI }\end{array}$ & $\begin{array}{l}14 \\
\text { VI }\end{array}$ & $\begin{array}{l}14 \\
\text { VI }\end{array}$ & $\begin{array}{c}1 \\
\text { VI }\end{array}$ & $\begin{array}{l}\text { 1B } \\
\text { VI }\end{array}$ & $\begin{array}{c}3 \\
\text { VI }\end{array}$ & $\begin{array}{l}1 \mathrm{~A} \\
\text { VI }\end{array}$ & $\begin{array}{l}8 \mathrm{~A} \\
\text { VI }\end{array}$ & $\begin{array}{l}8 \mathrm{~A} \\
\text { VI }\end{array}$ & $\begin{array}{l}3 \mathrm{~B} \\
\text { VI }\end{array}$ & $\begin{array}{c}4 \\
\text { VI }\end{array}$ & $\begin{array}{l}14 \\
\text { VI }\end{array}$ \\
\hline 9.92 & 54.51 & 54.34 & 51.70 & 51.78 & 51.27 & 51.27 & 51.92 & 52.23 & 52.20 & 56.08 & 48.22 & 49.39 & 50.90 & 53.25 & 60.22 & 59.96 & 52.16 & 51.15 & 49.06 & 51.78 & 60.53 & 59.90 & 54.20 & 51.28 & 51.71 \\
\hline 0.00 & 0.00 & & 00 & .00 & 0.00 & 0.00 & 0.00 & 0.00 & 0.00 & 0.00 & 0.00 & 0.00 & 0.00 & 0.00 & 0.00 & 0.00 & 0.00 & 0.00 & 0.00 & 0.00 & 0.00 & 0.00 & 0.00 & 0.00 & 0.00 \\
\hline 25.55 & 28.44 & 28.26 & 30.56 & 31.10 & 30.03 & 29.96 & 30.25 & 30.06 & 30.69 & 26.69 & 32.88 & 32.40 & 30.48 & 29.60 & 24.61 & 24.70 & 30.29 & 30.49 & 32.38 & 30.86 & 25.79 & 25.47 & 29.35 & 31.07 & 30.95 \\
\hline 0.16 & 0.26 & 0.26 & 0.33 & 36 & 0.27 & 0.31 & 0.27 & 0.20 & 0.25 & 0.20 & 0.07 & 0.07 & 0.07 & 0.14 & 0.20 & 0.21 & 0.08 & 0.12 & 0.06 & 0.21 & 0.20 & 0.20 & 0.12 & 0.10 & 0.12 \\
\hline 0.00 & $0 .($ & 0 & & & & & 0.00 & 0.00 & 0.00 & & 0.00 & 0.0 & 0.00 & 0.00 & 0.00 & 0.00 & 0.00 & 0.00 & 0.00 & 0.0 & 0.00 & 0.00 & 0.00 & 0.00 & 0.00 \\
\hline 0.04 & 0.06 & 0.05 & 0.11 & 0.11 & 0.09 & 0.11 & 0.11 & 0.08 & 0.09 & & 0.06 & 0.0 & 0.0 & 0.1 & & & 0.06 & 0.09 & 0.14 & 0. & 0. & 0.00 & 6 & 0.05 & 0.07 \\
\hline 6.77 & 10.73 & 10.75 & 13.87 & 13.80 & 13.45 & 13.42 & 13.45 & 13.07 & 13.02 & 9.32 & 16.19 & 15.4 & 13.70 & 10.9 & & 6.2 & 12.93 & 13.67 & 15.31 & 13.8 & 7. & 84 & 11. & 13.56 & 13.85 \\
\hline 8.37 & 21 & 27 & 10 & 3.94 & 4.42 & 4.34 & 4.37 & 4.49 & 4.43 & 6.98 & 2.51 & 2.8 & 3.81 & 5.08 & 8.05 & 8.44 & 4.24 & 3.94 & 3.26 & 3.1 & 6.6 & 7.15 & 4.90 & 3.81 & 3.72 \\
\hline 0.12 & 0.03 & 0.03 & 0.03 & 0.03 & 0.08 & 0.03 & 0.03 & 0.03 & 0.02 & 0.09 & 0.00 & 0.00 & 0.02 & 0.05 & 0.17 & 0.14 & 0.00 & 0.02 & 0.00 & 0.12 & 0.17 & 0.14 & 0.03 & 0.03 & 0.01 \\
\hline 00.93 & 99.24 & 98.96 & 100.70 & 101.12 & 99.61 & 99.44 & 100.40 & 100.16 & 100.70 & 99.43 & 99.93 & 100.18 & 99.04 & 99.22 & 99.42 & 99.76 & 99.76 & 99.48 & 100.211 & 100.42 & 100.39 & 99.70 & 100.53 & 99.90 & 100.43 \\
\hline 2.630 & 2.480 & 2.475 & 2.330 & 2.325 & 2.325 & 2.335 & 2.340 & 2.360 & 2.345 & 2.515 & 2.20 & & 2.33 & & & 2.66 & 2.370 & 2.335 & 2.225 & 2.350 & 2.708 & 2.687 & 2.435 & 2.330 & 2.340 \\
\hline & & & 0. & & 0.00 & 0.0 & 0.00 & & 0.0 & & & & & & & & & & & & & & & & \\
\hline 1.320 & 1.525 & .515 & 620 & .645 & 1.605 & 1.6 & 1.605 & 1.6 & 1.6 & & 1.7 & & 1.6 & & & & 1.6 & 1.640 & 1.730 & 1.650 & & 1.347 & 1.555 & 65 & 1.650 \\
\hline 0.005 & 0.010 & 0. & & & & & & & & & & & & & & & & & & & & & & & 0.005 \\
\hline 0.000 & 0.000 & 0.000 & 0.000 & 0.000 & 0.000 & 0.000 & 0.000 & 0.000 & 0.000 & 0.000 & 0.0 & & 0.0 & 0.0 & & & 0.0 & 0.000 & 0.000 & 0.000 & & 0.000 & & & 0.000 \\
\hline & & & & & & & & & & & & & & & & & & & & & & & & & 0.005 \\
\hline 0.320 & 0.520 & 0. & & & 0.65 & & & & & & & & & & & & & & & & & & & & 0.670 \\
\hline 0.710 & 0.460 & 0.465 & 0.355 & 0.345 & 0.390 & 0.3 & 0.380 & & & & 0.2 & & 0.3 & 0.4 & & 0.7 & 0.3 & 0.3 & 0.2 & 0.2 & & 0.6 & & 0.3 & 0.325 \\
\hline 0.010 & 0.005 & 0.005 & 0.005 & 0.005 & 0.005 & 0.005 & 0.005 & 0.005 & 0.000 & 0.005 & 0.000 & 0.000 & 0.000 & 0.005 & 0.010 & 0.010 & 0.000 & 0.000 & 0.000 & 0.010 & 0.010 & 0.008 & 0.005 & 0.005 & 0.000 \\
\hline 5.000 & 5.005 & 5.000 & 5.005 & 5.010 & 4.995 & 5.010 & 5.000 & 5.005 & 4.995 & 4.995 & 5.005 & 5.005 & 5.010 & 5.000 & 5.000 & 5.005 & 5.005 & 5.005 & 5.000 & 5.005 & 5.000 & 5.000 & 5.000 & 5.005 & 4.995 \\
\hline 30.8 & 52.8 & & & & & & & & & & & & & & & & & & & & & & & & 67.3 \\
\hline 68.3 & 46.7 & & & & 37.1 & 36.8 & 36.7 & & 38.1 & & 22.1 & & & & & & 3 & 34.2 & 27.7 & 29. & & 64.9 & & 33. & 32.7 \\
\hline 1.0 & 0.5 & 0.5 & 0.5 & 0.5 & 0.5 & 0.5 & 0.5 & 0.5 & 0.0 & 0.05 & 0.0 & 0.0 & 0.0 & 0.5 & 1.0 & 1.0 & 0.0 & 0.0 & 0.0 & 1.0 & 1.0 & 0.8 & 0.5 & 0.5 & 0.0 \\
\hline
\end{tabular}


Table 3. Representative microprobe analyses of clinopyroxene from Leg 118 plutonic rocks.

\begin{tabular}{|c|c|c|c|c|c|c|c|c|c|c|c|c|c|c|c|c|c|c|c|c|c|c|}
\hline $\begin{array}{c}\text { Core/section: } \\
\text { Interval }(\mathrm{cm}) \text { : } \\
\text { Piece: } \\
\text { Unit: }\end{array}$ & $\begin{array}{c}\text { 1D-1 } \\
90-92 \\
14 \\
\text { I }\end{array}$ & $\begin{array}{c}1 \mathrm{D}-1 \\
90-92 \\
14 \\
1\end{array}$ & $\begin{array}{c}13 \mathrm{R}-1 \\
102-105 \\
11 \mathrm{~B} \\
\text { II }\end{array}$ & $\begin{array}{c}14 \mathrm{R}-1 \\
26-29 \\
2 \mathrm{~A} \\
\text { II }\end{array}$ & $\begin{array}{c}14 \mathrm{R}-1 \\
26-29 \\
2 \mathrm{~A} \\
\text { II }\end{array}$ & $\begin{array}{c}18 \mathrm{R}-3 \\
37-40 \\
4 \\
\text { II }\end{array}$ & $\begin{array}{c}23 \mathrm{R}-3 \\
34-37 \\
2 \\
\text { II }\end{array}$ & $\begin{array}{c}25 \mathrm{R}-2 \\
5-7 \\
1 \mathrm{~A} \\
\text { II }\end{array}$ & $\begin{array}{c}38 \mathrm{R}-3 \\
58-64 \\
2 \\
\text { III }\end{array}$ & $\begin{array}{c}38 \mathrm{R}-3 \\
58-64 \\
2 \\
\text { III }\end{array}$ & $\begin{array}{c}42 \mathrm{R}-4 \\
115-123 \\
5 \mathrm{~A} \\
\text { III }\end{array}$ & $\begin{array}{c}42 \mathrm{R}-4 \\
115-123 \\
5 \mathrm{~A} \\
\text { III }\end{array}$ & $\begin{array}{c}44 \mathrm{R}-2 \\
0-4 \\
1 \mathrm{~A} \\
\text { III }\end{array}$ & $\begin{array}{c}44 \mathrm{R}-2 \\
115-122 \\
1 \mathrm{H} \\
\text { III }\end{array}$ & $\begin{array}{c}44 \mathrm{R}-3 \\
96-101 \\
4 \\
\text { III }\end{array}$ & $\begin{array}{c}45 \mathrm{R}-2 \\
19-24 \\
1 \mathrm{C} \\
\text { III }\end{array}$ & $\begin{array}{c}47 \mathrm{R}-2 \\
5-10 \\
1 \\
\text { IV }\end{array}$ & $\begin{array}{c}48 \mathrm{R}-3 \\
28-32 \\
1 \mathrm{C} \\
\text { IV }\end{array}$ & $\begin{array}{c}51 R-3 \\
110-114 \\
\text { IE } \\
\text { IV }\end{array}$ & $\begin{array}{c}52 \mathrm{R}-4 \\
12-25 \\
1 \\
\text { IV }\end{array}$ & $\begin{array}{c}54 R-4 \\
0-9 \\
1 \\
\text { IV }\end{array}$ & $\begin{array}{c}54 \mathrm{R}-4 \\
0-9 \\
1 \\
\text { IV }\end{array}$ \\
\hline $\begin{array}{l}\mathrm{SiO}_{2} \\
\mathrm{TiO}_{2} \\
\mathrm{Al}_{2} \mathrm{O}_{3} \\
\mathrm{Cr}_{2} \mathrm{O}_{3} \\
\mathrm{FeO} \\
\mathrm{MnO} \\
\mathrm{NiO} \\
\mathrm{MgO} \\
\mathrm{CaO} \\
\mathrm{Na}_{2} \mathrm{O} \\
\mathrm{K}_{2} \mathrm{O}\end{array}$ & $\begin{array}{r}51.52 \\
0.32 \\
1.81 \\
0.00 \\
10.03 \\
0.52 \\
0.00 \\
13.28 \\
21.41 \\
0.31 \\
0.02 \\
\end{array}$ & $\begin{array}{r}47.40 \\
0.69 \\
5.52 \\
0.00 \\
10.57 \\
0.45 \\
0.00 \\
13.39 \\
20.12 \\
0.69 \\
0.02\end{array}$ & $\begin{array}{r}50.62 \\
0.87 \\
2.87 \\
0.08 \\
8.10 \\
0.39 \\
0.00 \\
16.79 \\
19.27 \\
0.50 \\
0.02\end{array}$ & $\begin{array}{r}50.10 \\
0.71 \\
2.66 \\
0.00 \\
11.57 \\
0.51 \\
0.00 \\
14.19 \\
20.08 \\
0.40 \\
0.00\end{array}$ & $\begin{array}{r}51.82 \\
0.13 \\
0.68 \\
0.00 \\
8.21 \\
0.41 \\
0.00 \\
14.89 \\
22.78 \\
0.17 \\
0.00\end{array}$ & $\begin{array}{r}48.68 \\
0.94 \\
6.84 \\
0.25 \\
7.06 \\
0.27 \\
0.00 \\
17.04 \\
18.93 \\
1.11 \\
0.00\end{array}$ & $\begin{array}{r}50.05 \\
0.67 \\
2.30 \\
0.00 \\
11.45 \\
0.61 \\
0.00 \\
13.35 \\
21.10 \\
0.49 \\
0.00\end{array}$ & $\begin{array}{r}50.53 \\
0.55 \\
2.86 \\
0.00 \\
7.86 \\
0.33 \\
0.00 \\
15.86 \\
21.20 \\
0.42 \\
0.01\end{array}$ & $\begin{array}{r}50.77 \\
0.67 \\
2.13 \\
0.00 \\
12.42 \\
0.47 \\
0.00 \\
12.69 \\
20.62 \\
0.44 \\
0.00\end{array}$ & $\begin{array}{r}50.20 \\
0.63 \\
2.32 \\
0.00 \\
13.23 \\
0.49 \\
0.00 \\
12.93 \\
19.32 \\
0.46 \\
0.00\end{array}$ & $\begin{array}{r}50.40 \\
0.67 \\
2.29 \\
0.00 \\
11.68 \\
0.46 \\
0.00 \\
15.81 \\
19.09 \\
0.41 \\
0.00\end{array}$ & $\begin{array}{r}50.07 \\
0.75 \\
2.64 \\
0.00 \\
10.24 \\
0.43 \\
0.00 \\
14.58 \\
21.13 \\
0.47 \\
0.00\end{array}$ & $\begin{array}{r}51.33 \\
0.80 \\
2.57 \\
0.01 \\
10.96 \\
0.39 \\
0.00 \\
13.58 \\
20.28 \\
0.53 \\
0.00\end{array}$ & $\begin{array}{r}50.45 \\
0.75 \\
2.44 \\
0.00 \\
12.26 \\
0.37 \\
0.00 \\
12.79 \\
19.47 \\
0.50 \\
0.00\end{array}$ & $\begin{array}{r}52.19 \\
0.40 \\
3.38 \\
0.35 \\
6.50 \\
0.21 \\
0.00 \\
16.36 \\
20.90 \\
0.40 \\
0.00\end{array}$ & $\begin{array}{r}51.68 \\
0.54 \\
3.18 \\
0.36 \\
5.33 \\
0.49 \\
0.00 \\
16.58 \\
21.04 \\
0.34 \\
0.00\end{array}$ & $\begin{array}{r}51.17 \\
0.63 \\
1.93 \\
0.00 \\
11.89 \\
0.47 \\
0.00 \\
13.29 \\
19.75 \\
0.00 \\
0.00\end{array}$ & $\begin{array}{r}50.38 \\
0.81 \\
2.03 \\
0.00 \\
12.93 \\
0.62 \\
0.00 \\
13.10 \\
19.92 \\
0.45 \\
0.00\end{array}$ & $\begin{array}{r}51.18 \\
0.74 \\
2.31 \\
0.00 \\
12.86 \\
0.39 \\
0.00 \\
12.94 \\
18.95 \\
0.46 \\
0.00\end{array}$ & $\begin{array}{r}50.77 \\
0.64 \\
1.82 \\
0.02 \\
13.67 \\
0.60 \\
0.00 \\
12.57 \\
19.99 \\
0.40 \\
0.00\end{array}$ & $\begin{array}{r}51.03 \\
0.36 \\
1.18 \\
0.00 \\
16.22 \\
0.51 \\
0.00 \\
11.30 \\
18.95 \\
0.42 \\
0.00\end{array}$ & $\begin{array}{r}50.61 \\
0.41 \\
1.15 \\
0.00 \\
16.87 \\
0.70 \\
0.00 \\
11.54 \\
18.16 \\
0.49 \\
0.00\end{array}$ \\
\hline Total: & 99.22 & 98.85 & 99.51 & 100.22 & 99.09 & 101.12 & 100.02 & 99.62 & 100.21 & 99.58 & 100.81 & 100.31 & 100.45 & 99.03 & 100.69 & 99.54 & 99.13 & 100.24 & 99.83 & 100.48 & 99.97 & 99.93 \\
\hline $\begin{array}{l}\text { Si } \\
\text { Al IV }\end{array}$ & $\begin{array}{l}1.949 \\
0.051\end{array}$ & $\begin{array}{l}1.811 \\
0.189\end{array}$ & $\begin{array}{l}1.887 \\
0.113\end{array}$ & $\begin{array}{l}1.889 \\
0.111\end{array}$ & $\begin{array}{l}1.955 \\
0.030\end{array}$ & $\begin{array}{l}1.781 \\
0.219\end{array}$ & $\begin{array}{l}1.897 \\
0.103\end{array}$ & $\begin{array}{l}1.888 \\
0.112\end{array}$ & $\begin{array}{l}1.922 \\
0.078\end{array}$ & $\begin{array}{l}1.914 \\
0.086\end{array}$ & $\begin{array}{l}1.885 \\
0.101\end{array}$ & $\begin{array}{l}1.881 \\
0.117\end{array}$ & $\begin{array}{l}1.920 \\
0.080\end{array}$ & $\begin{array}{l}1.924 \\
0.076\end{array}$ & $\begin{array}{l}1.908 \\
0.092\end{array}$ & $\begin{array}{l}1.906 \\
0.094\end{array}$ & $\begin{array}{l}1.943 \\
0.055\end{array}$ & $\begin{array}{l}1.911 \\
0.089\end{array}$ & $\begin{array}{l}1.935 \\
0.065\end{array}$ & $\begin{array}{l}1.925 \\
0.075\end{array}$ & $\begin{array}{l}1.959 \\
0.041\end{array}$ & $\begin{array}{l}1.950 \\
0.050\end{array}$ \\
\hline $\begin{array}{l}\mathrm{Al} \mathrm{VI} \\
\mathrm{Fe}^{3+} \\
\mathrm{Ti} \\
\mathrm{Cr}\end{array}$ & $\begin{array}{l}0.0302 \\
0.0000 \\
0.0091 \\
0.0000\end{array}$ & $\begin{array}{l}0.0601 \\
0.0000 \\
0.0198 \\
0.0000\end{array}$ & $\begin{array}{l}0.0126 \\
0.0000 \\
0.0244 \\
0.0023\end{array}$ & $\begin{array}{l}0.0071 \\
0.0000 \\
0.0202 \\
0.0000\end{array}$ & $\begin{array}{l}0.0000 \\
0.0000 \\
0.0037 \\
0.0000\end{array}$ & $\begin{array}{l}0.0754 \\
0.0000 \\
0.0258 \\
0.0072\end{array}$ & $\begin{array}{l}0.0002 \\
0.0000 \\
0.0191 \\
0.0000\end{array}$ & $\begin{array}{l}0.0138 \\
0.0000 \\
0.0155 \\
0.0000\end{array}$ & $\begin{array}{l}0.0165 \\
0.0000 \\
0.0191 \\
0.0000\end{array}$ & $\begin{array}{l}0.0186 \\
0.0000 \\
0.0181 \\
0.0000\end{array}$ & $\begin{array}{l}0.0000 \\
0.0000 \\
0.0188 \\
0.0000\end{array}$ & $\begin{array}{l}0.0000 \\
0.0000 \\
0.0212 \\
0.0000\end{array}$ & $\begin{array}{l}0.0332 \\
0.0000 \\
0.0225 \\
0.0003\end{array}$ & $\begin{array}{l}0.0336 \\
0.0000 \\
0.0215 \\
0.0000\end{array}$ & $\begin{array}{l}0.0532 \\
0.0000 \\
0.0110 \\
0.0101\end{array}$ & $\begin{array}{l}0.0442 \\
0.0000 \\
0.0150 \\
0.0105\end{array}$ & $\begin{array}{l}0.0315 \\
0.0116 \\
0.0180 \\
0.0000\end{array}$ & $\begin{array}{l}0.0012 \\
0.0000 \\
0.0231 \\
0.0000\end{array}$ & $\begin{array}{l}0.0381 \\
0.0000 \\
0.0210 \\
0.0000\end{array}$ & $\begin{array}{l}0.0067 \\
0.0000 \\
0.0183 \\
0.0006\end{array}$ & $\begin{array}{l}0.0128 \\
0.0000 \\
0.0104 \\
0.0000\end{array}$ & $\begin{array}{l}0.0026 \\
0.0000 \\
0.0119 \\
0.0000\end{array}$ \\
\hline $\begin{array}{l}\mathrm{Ni} \\
\mathrm{Mg} \\
\mathrm{Fe}^{2+} \\
\mathrm{Mn}\end{array}$ & $\begin{array}{l}0.000 \\
0.748 \\
0.317 \\
0.0167\end{array}$ & $\begin{array}{l}0.000 \\
0.763 \\
0.338 \\
0.0146\end{array}$ & $\begin{array}{l}0.000 \\
0.933 \\
0.253 \\
0.0123\end{array}$ & $\begin{array}{l}0.000 \\
0.797 \\
0.365 \\
0.0163\end{array}$ & $\begin{array}{l}0.000 \\
0.837 \\
0.259 \\
0.0131\end{array}$ & $\begin{array}{l}0.000 \\
0.929 \\
0.216 \\
0.0084\end{array}$ & $\begin{array}{l}0.000 \\
0.754 \\
0.363 \\
0.0196\end{array}$ & $\begin{array}{l}0.000 \\
0.884 \\
0.246 \\
0.0104\end{array}$ & $\begin{array}{l}0.000 \\
0.716 \\
0.393 \\
0.0151\end{array}$ & $\begin{array}{l}0.000 \\
0.735 \\
0.421 \\
0.0158\end{array}$ & $\begin{array}{l}0.000 \\
0.881 \\
0.365 \\
0.0146\end{array}$ & $\begin{array}{l}0.000 \\
0.816 \\
0.321 \\
0.0137\end{array}$ & $\begin{array}{l}0.000 \\
0.758 \\
0.343 \\
0.0124\end{array}$ & $\begin{array}{l}0.000 \\
0.727 \\
0.391 \\
0.0120\end{array}$ & $\begin{array}{l}0.000 \\
0.891 \\
0.199 \\
0.0065\end{array}$ & $\begin{array}{l}0.000 \\
0.912 \\
0.165 \\
0.0153\end{array}$ & $\begin{array}{l}0.000 \\
0.752 \\
0.366 \\
0.0151\end{array}$ & $\begin{array}{l}0.000 \\
0.741 \\
0.410 \\
0.0199\end{array}$ & $\begin{array}{l}0.000 \\
0.730 \\
0.407 \\
0.0125\end{array}$ & $\begin{array}{l}0.000 \\
0.710 \\
0.433 \\
0.0193\end{array}$ & $\begin{array}{l}0.000 \\
0.647 \\
0.521 \\
0.0166\end{array}$ & $\begin{array}{l}0.000 \\
0.663 \\
0.544 \\
0.0228\end{array}$ \\
\hline $\begin{array}{l}\mathrm{Ca} \\
\mathrm{Na}\end{array}$ & $\begin{array}{l}0.868 \\
0.023 \\
\end{array}$ & $\begin{array}{l}0.823 \\
0.051 \\
\end{array}$ & $\begin{array}{l}0.769 \\
0.036 \\
\end{array}$ & $\begin{array}{l}0.811 \\
0.029 \\
\end{array}$ & $\begin{array}{l}0.921 \\
0.012 \\
\end{array}$ & $\begin{array}{l}0.742 \\
0.079 \\
\end{array}$ & $\begin{array}{l}0.858 \\
0.036 \\
\end{array}$ & $\begin{array}{l}0.849 \\
0.030 \\
\end{array}$ & $\begin{array}{l}0.836 \\
0.032 \\
\end{array}$ & $\begin{array}{l}0.789 \\
0.034 \\
\end{array}$ & $\begin{array}{l}0.765 \\
0.030\end{array}$ & $\begin{array}{l}0.851 \\
0.034 \\
\end{array}$ & $\begin{array}{l}0.813 \\
0.038 \\
\end{array}$ & $\begin{array}{l}0.796 \\
0.037 \\
\end{array}$ & $\begin{array}{l}0.819 \\
0.028 \\
\end{array}$ & $\begin{array}{l}0.831 \\
0.024 \\
\end{array}$ & $\begin{array}{l}0.803 \\
0.000 \\
\end{array}$ & $\begin{array}{l}0.809 \\
0.033 \\
\end{array}$ & $\begin{array}{l}0.768 \\
0.034\end{array}$ & $\begin{array}{l}0.812 \\
0.029 \\
\end{array}$ & $\begin{array}{l}0.780 \\
0.031\end{array}$ & $\begin{array}{l}0.750 \\
0.037 \\
\end{array}$ \\
\hline Total: & 4.012 & 4.070 & 4.043 & 4.047 & 4.032 & 0.082 & 4.050 & 4.049 & 4.028 & 4.032 & 4.061 & 4.056 & 4.020 & 4.018 & 4.018 & 4.017 & 3.995 & 4.037 & 4.009 & 4.030 & 4.019 & 4.030 \\
\hline $\begin{array}{l}\mathrm{Mg \#} \\
\text { Wo } \\
\text { En } \\
\text { Fs }\end{array}$ & $\begin{array}{c}0.702 \\
44.9 \\
38.7 \\
16.4\end{array}$ & $\begin{array}{l}0.693 \\
42.8 \\
39.7 \\
17.6\end{array}$ & $\begin{array}{l}0.787 \\
39.3 \\
47.7 \\
12.9\end{array}$ & $\begin{array}{l}0.686 \\
41.1 \\
40.4 \\
18.5\end{array}$ & $\begin{array}{l}0.764 \\
45.7 \\
41.5 \\
12.8\end{array}$ & $\begin{array}{l}0.811 \\
39.3 \\
49.2 \\
11.5\end{array}$ & $\begin{array}{l}0.675 \\
43.4 \\
38.2 \\
18.4\end{array}$ & $\begin{array}{c}0.782 \\
42.9 \\
44.7 \\
12.4\end{array}$ & $\begin{array}{l}0.646 \\
43.0 \\
36.8 \\
20.2\end{array}$ & $\begin{array}{l}0.636 \\
40.6 \\
37.8 \\
21.7\end{array}$ & $\begin{array}{l}0.707 \\
38.0 \\
43.8 \\
18.2\end{array}$ & $\begin{array}{l}0.717 \\
42.8 \\
41.1 \\
16.2\end{array}$ & $\begin{array}{l}0.689 \\
42.5 \\
39.6 \\
17.9\end{array}$ & $\begin{array}{c}0.65 \\
41.6 \\
38.0 \\
20.4\end{array}$ & $\begin{array}{c}0.818 \\
42.9 \\
46.7 \\
10.4\end{array}$ & $\begin{array}{c}0.847 \\
43.6 \\
47.8 \\
8.6\end{array}$ & $\begin{array}{l}0.673 \\
41.8 \\
39.2 \\
19.0\end{array}$ & $\begin{array}{l}0.644 \\
41.3 \\
37.8 \\
20.9\end{array}$ & $\begin{array}{l}0.642 \\
40.3 \\
38.3 \\
21.4\end{array}$ & $\begin{array}{l}0.621 \\
41.5 \\
36.3 \\
22.2\end{array}$ & $\begin{array}{l}0.554 \\
40.0 \\
33.2 \\
26.7\end{array}$ & $\begin{array}{l}0.549 \\
38.3 \\
33.9 \\
27.8\end{array}$ \\
\hline
\end{tabular}


Table 3 (continued).

\begin{tabular}{|c|c|c|c|c|c|c|c|c|c|c|c|c|c|c|c|c|c|c|c|}
\hline $\begin{array}{c}57 \mathrm{R}-3 \\
37-43 \\
1 \mathrm{C} \\
\mathrm{V}\end{array}$ & $\begin{array}{c}61 \mathrm{R}-1 \\
126-135 \\
5 \\
\mathrm{~V}\end{array}$ & $\begin{array}{c}64 \mathrm{R}-2 \\
74-84 \\
1 \mathrm{E} \\
\mathrm{V}\end{array}$ & $\begin{array}{c}64 \mathrm{R}-4 \\
105-112 \\
4 \\
\mathrm{~V}\end{array}$ & $\begin{array}{c}69 \mathrm{R}-2 \\
55-67 \\
2 \mathrm{~B} \\
\mathrm{~V}\end{array}$ & $\begin{array}{c}74 \mathrm{R}-5 \\
84-92 \\
3 \mathrm{~B} \\
\mathrm{~V}\end{array}$ & $\begin{array}{c}74 \mathrm{R}-6 \\
0-8 \\
1 \\
\mathrm{~V}\end{array}$ & $\begin{array}{c}75 \mathrm{R}-5 \\
45-53 \\
1 \mathrm{~B} \\
\mathrm{~V}\end{array}$ & $\begin{array}{c}79 \mathrm{R}-6 \\
105-111 \\
4 \\
\text { VI }\end{array}$ & $\begin{array}{c}79 \mathrm{R}-7 \\
104-109 \\
9 \\
\text { VI }\end{array}$ & $\begin{array}{c}79 \mathrm{R}-7 \\
104-109 \\
9 \\
\text { VI }\end{array}$ & $\begin{array}{c}80 \mathrm{R}-1 \\
117-123 \\
12 \\
\text { VI }\end{array}$ & $\begin{array}{c}80 \mathrm{R}-6 \\
121-129 \\
14 \\
\text { VI }\end{array}$ & $\begin{array}{c}81 \mathrm{R}-1 \\
130-132 \\
1 \\
\text { VI }\end{array}$ & $\begin{array}{c}82 \mathrm{R}-4 \\
12-20 \\
1 \mathrm{~B} \\
\text { VI }\end{array}$ & $\begin{array}{c}83 \mathrm{R}-6 \\
53-59 \\
3 \\
\mathrm{~V} 1\end{array}$ & $\begin{array}{c}84 \mathrm{R}-7 \\
13-23 \\
\text { ID } \\
\text { VI }\end{array}$ & $\begin{array}{c}86 \mathrm{R}-4 \\
64-70 \\
3 \mathrm{~B} \\
\mathrm{VI}\end{array}$ & $\begin{array}{c}86 \mathrm{R}-6 \\
28-38 \\
4 \\
\mathrm{VI}\end{array}$ & $\begin{array}{c}87 \mathrm{R}-6 \\
106-114 \\
14 \\
\text { VI }\end{array}$ \\
\hline 50.86 & 51.50 & 51.50 & 49.27 & 51.97 & 51.66 & 49.17 & 50.84 & 51.75 & 51.96 & 50.71 & 51.97 & 50.13 & 50.93 & 52.92 & 53.41 & 51.94 & 51.73 & 51.60 & 51.39 \\
\hline 0.62 & 0.53 & 0.45 & 0.50 & 0.40 & 0.55 & 0.49 & 0.48 & 0.38 & 0.91 & 0.78 & 0.38 & 0.38 & 0.42 & 0.41 & 0.38 & 0.58 & 0.56 & 1.54 & 0.83 \\
\hline 2.36 & 2.14 & 3.06 & 4.32 & 2.64 & 2.58 & 1.54 & 3.15 & 3.36 & 2.26 & 3.55 & 3.01 & 1.00 & 3.78 & 3.16 & 3.09 & 2.83 & 2.61 & 2.52 & 3.05 \\
\hline 0.04 & 0.10 & 0.26 & 0.21 & 0.17 & 0.11 & 0.00 & 0.12 & 0.88 & 0.06 & 0.98 & 0.95 & 0.00 & 0.29 & 0.38 & 0.25 & 0.04 & 0.07 & 0.05 & 0.10 \\
\hline 9.04 & 8.11 & 5.94 & 6.57 & 6.56 & 6.87 & 11.02 & 6.27 & 6.02 & 7.84 & 5.17 & 3.97 & 16.93 & 5.46 & 5.32 & 5.52 & 6.95 & 7.30 & 6.75 & 5.88 \\
\hline 0.37 & 0.32 & 0.21 & 0.27 & 0.25 & 0.29 & 0.50 & 0.36 & 0.19 & 0.30 & 0.23 & 0.17 & 1.43 & 0.20 & 0.17 & 0.17 & 0.30 & 0.29 & 0.24 & 0.25 \\
\hline 0.00 & 0.00 & 0.00 & 0.00 & 0.00 & 0.00 & 0.00 & 0.00 & 0.00 & 0.00 & 0.00 & 0.00 & 0.00 & 0.00 & 0.00 & 0.00 & 0.00 & 0.00 & 0.00 & 0.00 \\
\hline 16.86 & 16.60 & 18.15 & 17.06 & 17.96 & 17.74 & 14.64 & 17.61 & 18.88 & 16.29 & 15.23 & 17.52 & 12.65 & 18.22 & 17.24 & 17.37 & 16.16 & 16.73 & 14.53 & 16.69 \\
\hline 18.09 & 20.23 & 19.82 & 21.48 & 19.80 & 20.18 & 21.40 & 20.56 & 17.17 & 20.62 & 21.65 & 21.76 & 17.48 & 20.04 & 20.51 & 19.90 & 21.17 & 20.39 & 21.75 & 20.52 \\
\hline 0.44 & 0.38 & 0.42 & 0.43 & 0.38 & 0.41 & 0.55 & 0.35 & 0.38 & 0.71 & 0.93 & 0.67 & 0.07 & 0.38 & 0.41 & 0.35 & 0.36 & 0.56 & 0.47 & 0.39 \\
\hline 0.00 & 0.00 & 0.00 & 0.00 & 0.00 & 0.00 & 0.00 & 0.00 & 0.00 & 0.00 & 0.00 & 0.00 & 0.00 & 0.00 & 0.00 & 0.00 & 0.00 & 0.00 & 0.00 & 0.00 \\
\hline 98.68 & 99.91 & 99.81 & 100.11 & 100.13 & 100.39 & 99.31 & 99.74 & 99.01 & 100.95 & 99.23 & 100.40 & 100.07 & 99.72 & 100.52 & 100.44 & 100.33 & 100.24 & 99.45 & 99.10 \\
\hline 1.911 & 1.913 & 1.893 & 1.827 & 1.908 & 1.898 & 1.882 & 1.880 & 1.903 & 1.910 & 1.886 & 1.896 & 1.932 & 1.872 & 1.922 & 1.937 & 1.912 & 1.908 & 1.919 & $\begin{array}{l}1.904 \\
0.096\end{array}$ \\
\hline 0.089 & 0.087 & 0.107 & 0.173 & 0.092 & 0.102 & 0.070 & 0.120 & 0.097 & 0.090 & 0.114 & 0.104 & 0.045 & 0.128 & 0.078 & 0.061 & 0.088 & 0.092 & 0.080 & 0.096 \\
\hline 0.0157 & 0.0062 & 0.0252 & 0.0160 & 0.0218 & 0.0100 & 0.0000 & 0.0168 & 0.0487 & 0.0077 & 0.0415 & 0.0250 & 0.0000 & 0.0353 & 0.0578 & 0.0710 & 0.0347 & 0.0216 & 0.0307 & 0.0376 \\
\hline 0.0000 & 0.0000 & 0000 & 0000 & 0.0000 & 0.0000 & 0.0000 & 0.0000 & 0.0000 & 0000 & 0.000 & 0.0000 & 0.00 & 0.0000 & 0.0000 & 0.0139 & 0.0000 & 0.0000 & 0.0035 & 0.0000 \\
\hline 0.0175 & 0.0148 & 0.0124 & 039 & 0.0111 & 0.0152 & 0.01 & 0.0134 & 0.01 & 0.025 & 0.02 & & & & 0.0112 & & 0.01 & 0.01 & & 0.0231 \\
\hline 0.0012 & 0.0029 & 0.0076 & 0.0062 & 0.0049 & 0.0032 & 0.0000 & 0.0035 & 0.0256 & 0.0017 & 0.0288 & 0.0274 & 0.0000 & 0.0085 & 0.0109 & 0.0072 & 0.0012 & 0.0020 & 0.0015 & 0.0029 \\
\hline 0.000 & 0.000 & 0.000 & 0.000 & 0.000 & 0.000 & 0.000 & 0.000 & 0.000 & 0.000 & 0.000 & 0.000 & 0.000 & 0.000 & 0.000 & 0.000 & 0.000 & 0.000 & 0.000 & 0.000 \\
\hline 0.944 & & 0.995 & & & & & & & & & & & & 0.93 & & & & & 0.922 \\
\hline 0.284 & 0.252 & 0.183 & 0.204 & 0.202 & 0.211 & 0.353 & 0.194 & 0.185 & 0.241 & 0.160 & 0.121 & 0.546 & 0.168 & 0.161 & 0.154 & 0.214 & 0.225 & 0.207 & 0.182 \\
\hline 0.0118 & 0.0101 & 0.0065 & 0.0085 & 0.0077 & 0.0090 & 0.0162 & 0.0113 & 0.0059 & 0.0093 & 0.0072 & 0.0053 & 0.0467 & 0.0062 & 0.0052 & 0.0052 & 0.0094 & 0.0090 & 0.0076 & 0.0078 \\
\hline $\begin{array}{l}0.728 \\
0.032\end{array}$ & $\begin{array}{l}0.805 \\
0.027\end{array}$ & $\begin{array}{l}0.781 \\
0.030\end{array}$ & $\begin{array}{l}0.854 \\
0.031\end{array}$ & $\begin{array}{l}0.779 \\
0.027\end{array}$ & $\begin{array}{l}0.795 \\
0.029\end{array}$ & $\begin{array}{l}0.878 \\
0.041\end{array}$ & $\begin{array}{l}0.815 \\
0.025\end{array}$ & $\begin{array}{l}0.677 \\
0.027\end{array}$ & $\begin{array}{l}0.812 \\
0.051\end{array}$ & $\begin{array}{l}0.862 \\
0.067\end{array}$ & $\begin{array}{l}0.850 \\
0.047\end{array}$ & $\begin{array}{l}0.722 \\
0.005\end{array}$ & $\begin{array}{l}0.790 \\
0.027\end{array}$ & $\begin{array}{l}0.798 \\
0.029\end{array}$ & $\begin{array}{l}0.773 \\
0.025\end{array}$ & $\begin{array}{l}0.835 \\
0.026\end{array}$ & $\begin{array}{l}0.806 \\
0.040\end{array}$ & $\begin{array}{l}0.867 \\
0.034\end{array}$ & $\begin{array}{l}0.815 \\
0.028\end{array}$ \\
\hline 4.035 & 4.038 & 4.040 & 4.077 & 4.036 & 4.044 & 4.089 & 4.050 & 4.014 & 4.041 & 4.033 & 4.039 & 4.037 & 4.044 & 0.023 & 3.996 & 4.023 & 0.070 & 3.099 & 4.018 \\
\hline 0.768 & 0.785 & 0.845 & 0.822 & 0.830 & 0.821 & 0.703 & 0.833 & 0.849 & 0.787 & 0.840 & 0.887 & 0.571 & 0.856 & 0.853 & 0.859 & 0.805 & 0.803 & 0.796 & 0.835 \\
\hline 37.2 & 40.7 & 39.9 & 42.7 & 39.7 & 40.2 & 42.5 & 41.2 & 35.7 & 41.7 & 46.2 & 44.2 & 36.2 & 40.4 & 42.2 & 41.4 & 43.1 & 41.3 & 46.1 & 42.5 \\
\hline 48.2 & 46.5 & 50.8 & 47.1 & 50.1 & 49.1 & 40.4 & 49.0 & 54.6 & 45.9 & 45.2 & 49.5 & 36.5 & 51.0 & 49.3 & 50.3 & 45.8 & 47.2 & 42.9 & 48.1 \\
\hline 14.5 & 12.8 & 9.3 & 10.2 & 10.3 & 10.7 & 17.1 & 9.8 & 9.7 & 12.4 & 8.6 & 6.3 & 27.4 & 8.6 & 8.5 & 8.2 & 11.1 & 11.5 & 11.0 & 9.5 \\
\hline
\end{tabular}


Table 4. Representative microprobe analyses of orthopyroxene from Leg 118 plutonic rocks.

\begin{tabular}{|c|c|c|c|c|c|}
\hline $\begin{array}{c}\text { Core/section: } \\
\text { Interval: }(\mathrm{cm}) \\
\text { Piece: } \\
\text { Unit: }\end{array}$ & $\begin{array}{c}44 \mathrm{R}-2 \\
115-122 \\
1 \mathrm{H} \\
\text { III }\end{array}$ & $\begin{array}{c}48 \mathrm{R}-4 \\
32-37 \\
3 \\
\text { IV }\end{array}$ & $\begin{array}{c}74 \mathrm{R}-6 \\
0-8 \\
1 \\
\mathrm{~V}\end{array}$ & $\begin{array}{c}80 \mathrm{R}-6 \\
121-129 \\
14 \\
\text { VI }\end{array}$ & $\begin{array}{c}85 \mathrm{R}-7 \\
0-5 \\
1 \mathrm{~A} \\
\text { VI }\end{array}$ \\
\hline $\begin{array}{l}\mathrm{SiO}_{2} \\
\mathrm{TiO}_{2} \\
\mathrm{Al}_{2} \mathrm{O}_{3} \\
\mathrm{Cr}_{2} \mathrm{O}_{3} \\
\mathrm{FeO} \\
\mathrm{MnO} \\
\mathrm{NiO} \\
\mathrm{MgO} \\
\mathrm{CaO} \\
\mathrm{Na}_{2} \mathrm{O} \\
\mathrm{K}_{2} \mathrm{O}\end{array}$ & $\begin{array}{r}52.71 \\
0.34 \\
1.04 \\
0.00 \\
21.87 \\
0.63 \\
0.00 \\
21.54 \\
1.73 \\
0.02 \\
0.00\end{array}$ & $\begin{array}{r}51.78 \\
0.22 \\
0.70 \\
0.00 \\
24.28 \\
0.98 \\
0.00 \\
19.61 \\
2.42 \\
0.03 \\
0.00\end{array}$ & $\begin{array}{r}50.87 \\
0.41 \\
0.77 \\
0.00 \\
22.60 \\
0.82 \\
0.00 \\
22.01 \\
3.84 \\
0.04 \\
0.00\end{array}$ & $\begin{array}{r}49.36 \\
0.15 \\
0.64 \\
0.00 \\
30.29 \\
1.32 \\
0.00 \\
16.68 \\
1.30 \\
0.03 \\
0.00\end{array}$ & $\begin{array}{r}55.37 \\
0.35 \\
1.16 \\
0.06 \\
10.47 \\
0.30 \\
0.00 \\
31.14 \\
0.76 \\
0.00 \\
0.00\end{array}$ \\
\hline Total: & 99.98 & 100.02 & 101.36 & 99.77 & 99.61 \\
\hline $\begin{array}{l}\text { Si } \\
\text { Al IV }\end{array}$ & $\begin{array}{l}1.970 \\
0.030\end{array}$ & $\begin{array}{l}1.965 \\
0.031\end{array}$ & $\begin{array}{l}1.904 \\
0.034\end{array}$ & $\begin{array}{l}1.938 \\
0.030\end{array}$ & $\begin{array}{l}1.958 \\
0.042\end{array}$ \\
\hline $\begin{array}{l}\mathrm{Al} \mathrm{VI} \\
\mathrm{Fe}^{3+} \\
\mathrm{Ti} \\
\mathrm{Cr}\end{array}$ & $\begin{array}{l}0.0159 \\
0.0022 \\
0.0096 \\
0.0000\end{array}$ & $\begin{array}{l}0.0000 \\
0.0000 \\
0.0063 \\
0.0000\end{array}$ & $\begin{array}{l}0.0000 \\
0.0000 \\
0.0116 \\
0.0000\end{array}$ & $\begin{array}{l}0.0000 \\
0.0000 \\
0.0045 \\
0.0000\end{array}$ & $\begin{array}{l}0.0068 \\
0.0000 \\
0.0093 \\
0.0017\end{array}$ \\
\hline $\begin{array}{l}\mathrm{Ni} \\
\mathrm{Mg} \\
\mathrm{Fe}^{2+} \\
\mathrm{Mn}\end{array}$ & $\begin{array}{l}0.000 \\
1.200 \\
0.681 \\
0.020\end{array}$ & $\begin{array}{l}0.000 \\
1.109 \\
0.771 \\
0.031\end{array}$ & $\begin{array}{l}0.000 \\
1.229 \\
0.708 \\
0.026\end{array}$ & $\begin{array}{l}0.000 \\
0.977 \\
0.995 \\
0.044\end{array}$ & $\begin{array}{l}0.000 \\
1.642 \\
0.310 \\
0.009\end{array}$ \\
\hline $\begin{array}{l}\mathrm{Ca} \\
\mathrm{Na}\end{array}$ & $\begin{array}{l}0.069 \\
0.001\end{array}$ & $\begin{array}{l}0.098 \\
0.002\end{array}$ & $\begin{array}{l}0.154 \\
0.003\end{array}$ & $\begin{array}{l}0.055 \\
0.002\end{array}$ & $\begin{array}{l}0.029 \\
0.000\end{array}$ \\
\hline Total: & 3.998 & 4.014 & 4.069 & 4.044 & 4.007 \\
\hline $\begin{array}{l}\text { Mg\# } \\
\text { Wo } \\
\text { En } \\
\text { Fs }\end{array}$ & $\begin{array}{l}0.638 \\
3.5 \\
61.5 \\
34.9\end{array}$ & $\begin{array}{c}0.590 \\
5.0 \\
56.1 \\
39.0\end{array}$ & $\begin{array}{l}0.634 \\
7.3 \\
58.8 \\
33.9\end{array}$ & $\begin{array}{l}0.495 \\
2.7 \\
48.2 \\
49.1\end{array}$ & $\begin{array}{l}0.841 \\
1.5 \\
82.9 \\
15.6\end{array}$ \\
\hline
\end{tabular}

and $\mathrm{Ti}$ vs. $\mathrm{Al}(\mathrm{iv})$ relationships of the pyroxenes (Fig. 6A and $6 \mathrm{~B}$, respectively). In the diagram of $\mathrm{Ti}$ vs. $\mathrm{Cr}$, clinopyroxenes from troctolites of Unit VI plot within or near the oceanic ultramafic cumulate field (comprising troctolites from the Garrett Fracture Zone; Hébert et al., 1983). Clinopyroxenes from Section 118-735B-80R-6 (Unit VI) plot in the oceanic gabbro field and were formed by crystallization from an evolved Ti-rich, Cr-poor liquid. Clinopyroxenes from Units V and IV plot in the same field. Clinopyroxenes from Unit III plot on a slightly different line of evolution, suggesting that these rocks were in equilibrium with a melt of different composition. However, the lines of evolution are parallel. In Figure 6B, a positive correlation of $\mathrm{Al}(\mathrm{iv}) \mathrm{vs}$. Ti takes place in Leg 118 clinopyroxenes. Only three analyses plot in the field of metamorphic clinopyroxenes defined by Mével (1987). This correlation illustrates early $\mathrm{Ti}$ and $\mathrm{Al}$ partitioning into clinopyroxene before ilmenite and plagioclase became important liquidus phases. In addition, experimental results demonstrate the pressure-dependence of $\mathrm{Al}$ that partitioned into clinopyroxene (Elthon, 1987; Colson and Gust, 1989).

\section{MINERAL COVARIATION}

Evolutionary trends of co-crystallizing phases are useful for characterizing igneous suites (Longhi, 1982). Figure 7 presents the variation of An in plagioclase vs. Fo in olivine (Fig. 7A) and $\mathrm{An}$ vs. $\mathrm{Mg} /\left(\mathrm{Mg}+\mathrm{Fe}^{2+}\right)$ in clinopyroxene (Fig. 7B). In both diagrams, Leg 118 gabbros plot within the previously defined spectrum for oceanic cumulates, and nearly perfect correlation exists among the various parameters. Covariation arrays for gabbros from the Garrett Fracture Zone and the Mid-Cayman Rise span a smaller range than do Leg 118 gabbros. The An content of plagioclase from the Mid-Cayman Rise may decrease more rapidly than it does in plagioclase from other sequences. Leg 118 gabbros extend the mineral covariation arrays to more evolved compositions than previously known for oceanic cumulates. The most primitive Leg 118 gabbros (those of Unit VI) plot in the primitive part of the array in Figure 7, but are somewhat more evolved than the most primitive cumulates recognized so far (Hébert, 1985). One interval within Unit VI (Section 118-735B-80R-6) contains evolved minerals that lie along the covariation trends extending from the most magnesian cumulates. This suggests that if these evolved gabbros were formed by differentiation of mixed magmas, the liquids involved were derived from similar melts. The entire sequence of gabbros was derived from liquids having high initial $\mathrm{Ca} /(\mathrm{Ca}+\mathrm{Na})$ ratios and $\mathrm{Mg} /(\mathrm{Mg}+$ $\mathrm{Fe}^{2+}$ ), which evolved toward $\mathrm{Na}$ - and $\mathrm{Fe}$-rich end-members.

At various stages of evolution, primitive and more fractionated melts were mixed, and these mixed liquids then evolved further to form strongly Fe-enriched melts. Two major fractionation trends can be recognized (Fig. 7): one parallel to the normal trend for oceanic cumulates, the other parallel to the trends of Mid-Cayman Rise gabbros. Fractional crystallization, removal of intercumulus liquids, and mixing of cotectic crystals and liquids are necessary to explain the linear covariation trends of oceanic cumulates (Longhi, 1982). These processes are thought to occur at generally moderate pressures (5-10 kb) (Grove and Bryan, 1983; Elthon, 1987) because the An/Fo molecular ratio is near unity, in agreement with experimental cotectic crystallization (Roeder and

Table 5. Representative microprobe analyses of ilmenite from Leg 118 plutonic rocks.

\begin{tabular}{|c|c|c|c|c|c|c|c|}
\hline $\begin{array}{l}\text { Core/section: } \\
\text { Interval }(\mathrm{cm}): \\
\text { Piece: } \\
\text { Unit: }\end{array}$ & $\begin{array}{c}44 \mathrm{R}-2 \\
115-122 \\
1 \mathrm{H} \\
\text { III }\end{array}$ & $\begin{array}{c}48 \mathrm{R}-2 \\
98-102 \\
8 \\
\text { IV }\end{array}$ & $\begin{array}{c}48 \mathrm{R}-3 \\
28-32 \\
1 \mathrm{C} \\
\text { IV }\end{array}$ & $\begin{array}{l}50 \mathrm{R}-3 \\
91-98 \\
\text { 1D } \\
\text { IV }\end{array}$ & $\begin{array}{c}51 \mathrm{R}-1 \\
79-84 \\
\text { 1D } \\
\text { IV }\end{array}$ & $\begin{array}{c}51 \mathrm{R}-3 \\
110-114 \\
1 \mathrm{E} \\
\text { IV }\end{array}$ & $\begin{array}{c}86 \mathrm{R}-1 \\
94-101 \\
8 \mathrm{~A} \\
\text { IV }\end{array}$ \\
\hline $\begin{array}{l}\mathrm{FeO} \\
\mathrm{MnO} \\
\mathrm{TiO}_{2} \\
\mathrm{MgO}\end{array}$ & $\begin{array}{r}46.68 \\
1.77 \\
50.61 \\
0.17\end{array}$ & $\begin{array}{r}47.60 \\
1.12 \\
50.77 \\
0.73\end{array}$ & $\begin{array}{r}45.73 \\
1.45 \\
50.07 \\
1.84\end{array}$ & $\begin{array}{r}45.98 \\
1.24 \\
51.23 \\
2.06\end{array}$ & $\begin{array}{r}48.93 \\
1.05 \\
49.67 \\
1.13\end{array}$ & $\begin{array}{r}46.56 \\
0.66 \\
51.08 \\
1.45\end{array}$ & $\begin{array}{r}45.90 \\
1.19 \\
50.24 \\
1.21\end{array}$ \\
\hline Total: & 99.23 & 100.22 & 99.09 & 100.51 & 100.78 & 99.75 & 98.54 \\
\hline $\begin{array}{l}\mathrm{Fe} \\
\mathrm{Mn} \\
\mathrm{Ti} \\
\mathrm{Mg}\end{array}$ & $\begin{array}{l}0.977 \\
0.037 \\
0.953 \\
0.006\end{array}$ & $\begin{array}{l}1.017 \\
0.024 \\
0.976 \\
0.028\end{array}$ & $\begin{array}{l}0.948 \\
0.030 \\
0.934 \\
0.068\end{array}$ & $\begin{array}{l}0.980 \\
0.027 \\
0.982 \\
0.079\end{array}$ & $\begin{array}{l}1.065 \\
0.023 \\
0.972 \\
0.044\end{array}$ & $\begin{array}{l}0.978 \\
0.014 \\
0.965 \\
0.054\end{array}$ & $\begin{array}{l}0.973 \\
0.025 \\
0.927 \\
0.044\end{array}$ \\
\hline Total: & 1.974 & 2.045 & 1.981 & 2.068 & 2.104 & 2.010 & 1.969 \\
\hline
\end{tabular}


Table 6. Calculated magma compositions starting from minerals accumulated in troctolites of lithologic Unit VI, Leg 118.

\begin{tabular}{lrrrr}
\hline \multirow{2}{*}{$\begin{array}{c}\text { Oxides } \\
\text { wt\% }\end{array}$} & \multicolumn{4}{c}{ Cores } \\
\cline { 2 - 5 } & $79 \mathrm{R}-6$ & $80 \mathrm{R}-1$ & $82 \mathrm{R}-4$ & $83 \mathrm{R}-6$ \\
\hline $\mathrm{SiO}_{2}$ & 47.88 & 49.32 & 48.80 & 48.87 \\
$\mathrm{TiO}_{2}$ & 1.69 & 1.23 & 1.48 & 1.63 \\
$\mathrm{Al}_{2} \mathrm{O}_{3}$ & 13.47 & 12.68 & 12.34 & 13.14 \\
$\mathrm{FeO}$ & 14.29 & 12.88 & 14.23 & 12.23 \\
$\mathrm{MnO}$ & 0.30 & 0.26 & 0.29 & 0.26 \\
$\mathrm{MgO}$ & 10.01 & 10.53 & 9.95 & 10.57 \\
$\mathrm{CaO}$ & 9.81 & 10.36 & 9.20 & 9.78 \\
$\mathrm{Na}{ }_{2} \mathrm{O}$ & 2.53 & 2.69 & 3.21 & 3.49 \\
${ }^{\mathrm{a}} \mathrm{P}_{2} \mathrm{O}_{5}$ & 0.17 & 0.12 & 0.15 & 0.16 \\
$\mathrm{Total}_{\mathrm{Tr}}$ & 100.18 & 100.07 & 100.17 & 100.13 \\
$\mathrm{Cr}(\mathrm{ppm})$ & 310 & 431 & 179 & 195 \\
$\mathrm{Ni}(\mathrm{ppm})$ & 24 & 54 & 18 & 24 \\
$\mathrm{Mg \#}$ & 0.35 & 0.39 & 0.35 & 0.40 \\
$\mathrm{CaO} / \mathrm{Na}_{2} \mathrm{O}$ & 3.87 & 3.85 & 2.48 & 2.80 \\
$\mathrm{CaO} / \mathrm{Al}_{2} \mathrm{O}_{3}$ & 0.73 & 0.82 & 0.75 & 0.75 \\
\hline
\end{tabular}

${ }^{a}$ Calculated after Morel (1979).

Emslie, 1970; Drake, 1976; Longhi, 1982). However, the presence of pigeonite and olivine plus plagioclase assemblages in some Leg 118 gabbros suggests an upper limit of about $8 \mathrm{~kb}$ for crystallization (Fujii and Bougault, 1983; Elthon and Scarfe, 1984). The oceanic mineral covariation array is a useful means of distinguishing MORB-related ophiolites (e.g.,
North Apennine ophiolites, Hébert et al., 1989) from arcrelated ophiolites (e.g, Troodos ophiolite, Hébert and Laurent, in press).

\section{COMPOSITION OF MELTS IN EQUILIBRIUM WITH MAGNESIAN CUMULATES}

$\mathrm{Fe}-\mathrm{Mg}$ partitioning between olivine and the melt was largely independent of temperature, pressure, and composition of the phases present (Roeder and Emslie, 1970), at least for pressures of less than $10 \mathrm{~kb}$ (Ulmer, 1989). Using a KD value of 0.27 , we have calculated $\mathrm{FeO}^{*} / \mathrm{MgO}$ ratios of the liquids in equilibrium with cumulus olivine. $\mathrm{FeO} * / \mathrm{MgO}$ ratios of these liquids vary from 1.1 to 2.7 (Fig. 8), a range similar to that measured from Pacific MOR lavas (Pallister and Hopson, 1981). Mid-Atlantic Ridge lavas exhibit more primitive ratios, some as low as 0.6 (Fig. 8). Making use of olivine-, clinopyroxene-, and plagioclase-liquid partition coefficients (Roeder and Emslie, 1970; Grove and Bryan, 1983; Drake, 1976), we calculated the predicted compositions of liquids capable of generating the troctolite of Unit VI (Table 6; see details in Hébert and Laurent, in press). Plots of calculated mol\% $\mathrm{MgO}$ and $\mathrm{FeO}$ in the liquids vs. the microprobe analyses of equilibrated olivine (Fig. 9) suggest temperatures of formation of the cumulates between $1250^{\circ}$ and $1300^{\circ} \mathrm{C}$. Estimated temperatures of formation do not vary with depth for this unit. $\mathrm{CaO} / \mathrm{Al}_{2} \mathrm{O}_{3}$ ratios are near those calculated from MORB liquids at $1280^{\circ} \mathrm{C}($ McKenzie and Bickle, 1988) and are higher than those calculated for primitive liquids of the Mid-Cayman

A
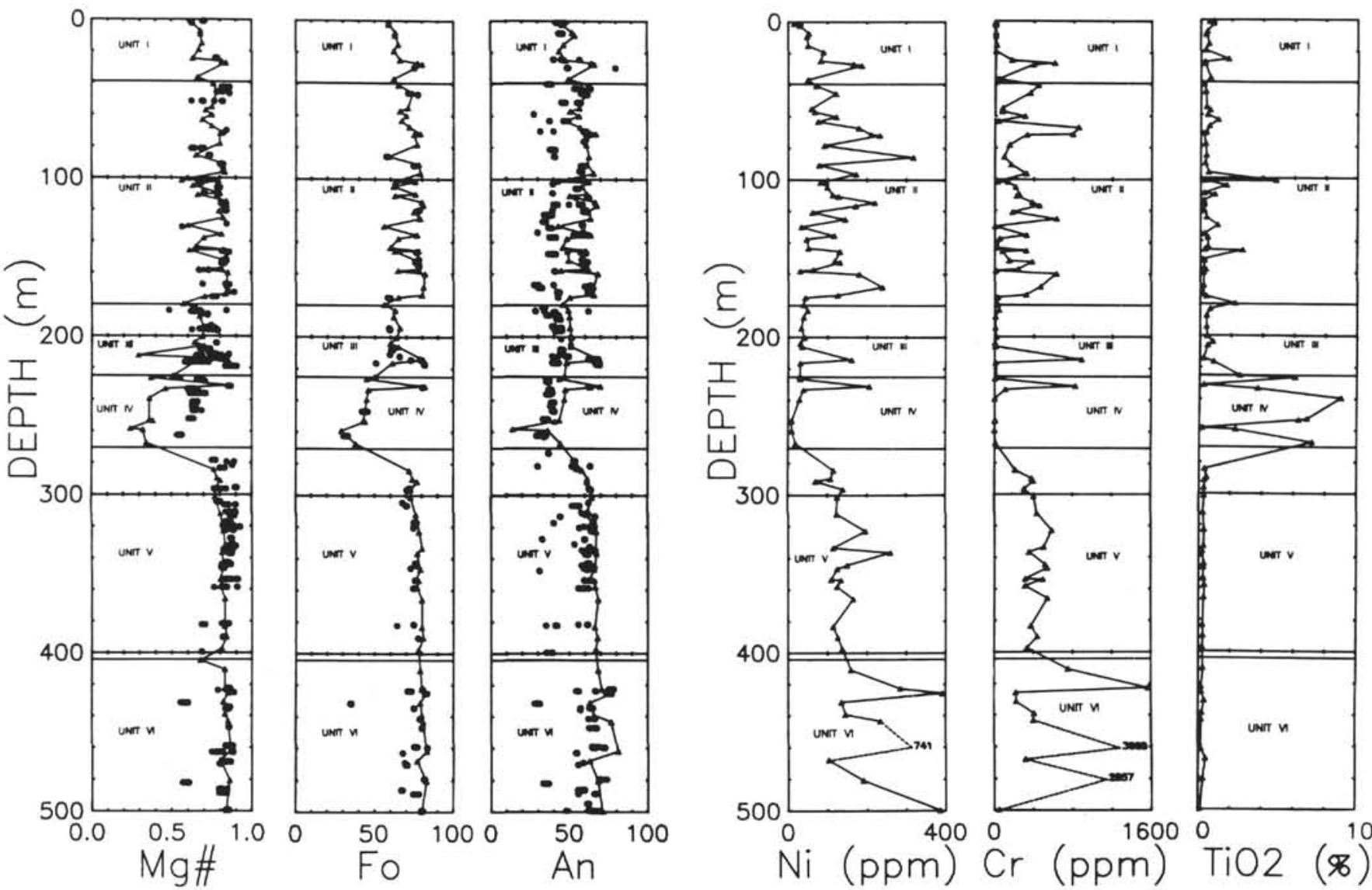

Figure 2. Geochemical stratigraphic logs. A. Normative $\mathrm{Mg}$, Fo in olivine, and An in plagioclase. B. $\mathrm{Ni}, \mathrm{Cr}$, and $\mathrm{TiO} \mathrm{O}_{2}$ variations. Continuous lines are connected data from Leg 118 shipboard geochemical analyses. Dots are microprobe analyses of minerals. Mg\# is calculated from clinopyroxene. 

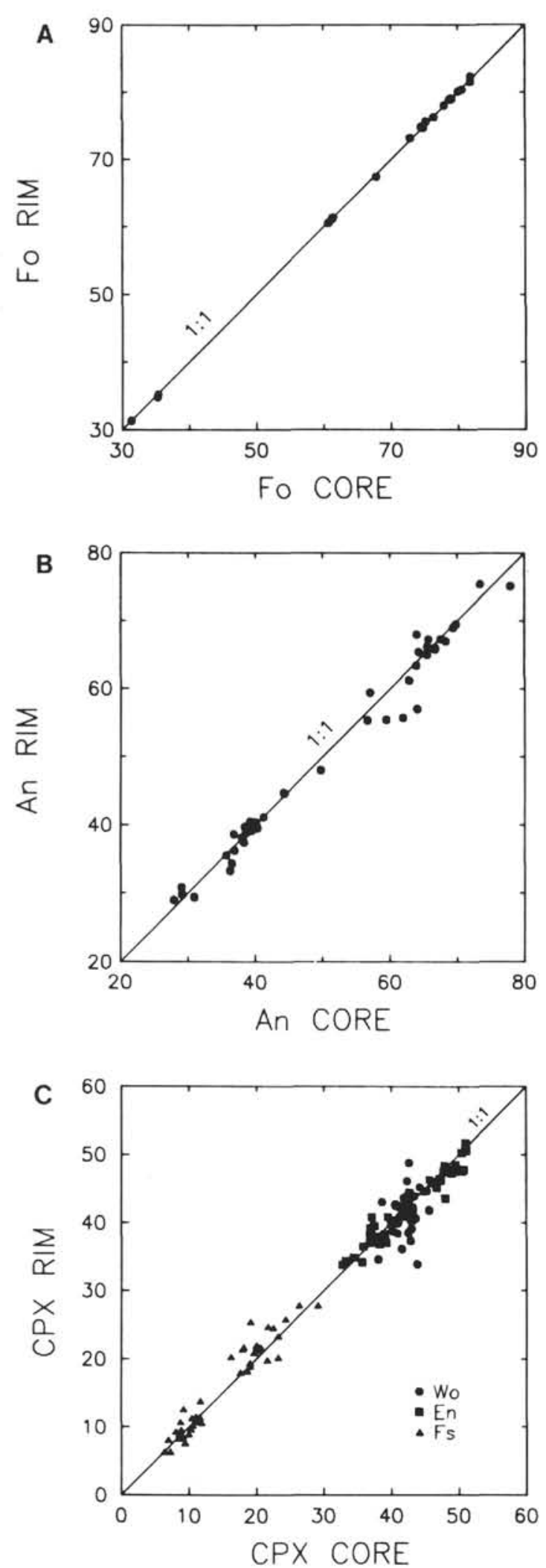

Figure 3. Binary diagrams of analyzed rims and cores of single phase. A. Olivine. B. Plagioclase. C. Clinopyroxene. Scales are units of molecular proportions. The line, 1:1, is shown for reference.
Rise (Elthon, 1987). The $\mathrm{Mg} /\left(\mathrm{Mg}+\mathrm{Fe}^{2+}\right)$ of the calculated liquids are not comparable to those of the most primitive glasses reported from the FAMOUS area (Stakes et al., 1984; Reid et al., 1989). $\mathrm{CaO} / \mathrm{Al}_{2} \mathrm{O}_{3}$ ratios are low, varying from 0.73 to 0.83 , which suggests that clinopyroxene largely fractionated from more primitive melt compositions (Bence et al., 1979; Reid et al., 1989). Comparisons with some Mid-Atlantic Ridge primitive liquids (Bryan, 1983; Reid et al., 1989) indicate lower $\mathrm{TiO}_{2}$ contents at a given $\mathrm{Mg} /\left(\mathrm{Mg}+\mathrm{Fe}^{2+}\right)$ for Atlantis II Fracture Zone magmas (0.60-0.90 wt\% $\mathrm{TiO}_{2}$ for MAR liquids). Diagrams of $\mathrm{Mg} /\left(\mathrm{Mg}+\mathrm{Fe}^{2+}\right)$ vs. $\mathrm{TiO}_{2}$ (not shown here) suggest that the calculated liquids of Table 6 are the products of extensive fractional crystallization because they plot away from the most primitive glasses (Stakes et al., 1984; Hekinian et al., 1976). Ulmer's experimental results (1989) indicated low pressure fractionation of the liquids. For instance, calculated liquids for Leg 118 gabbros are chemically equivalent to those formed by melting of nonrefractory sources at pressures of about $9 \mathrm{~kb}$ (Presnall et al., 1979). However, the source of the calculated liquids for Leg 118 gabbros is difficult to determine if magma mixing occurred. Magma mixing can explain local (Unit IV) high $\mathrm{TiO}_{2}$ and $\mathrm{P}_{2} \mathrm{O}_{5}$ values for a given $\mathrm{Mg} /\left(\mathrm{Mg}+\mathrm{Fe}^{2+}\right)$ of the liquid. If such mixing occurred, it is virtually impossible to calculate the primitive melt composition because both $\mathrm{TiO}_{2}$ and $\mathrm{Mg} /(\mathrm{Mg}+$ $\mathrm{Fe}^{2+}$ ) resulted from mixing of unknown volumes of melts having variable $\mathrm{TiO}_{2}$ and $\mathrm{Mg} /\left(\mathrm{Mg}+\mathrm{Fe}^{2+}\right)$.

\section{NATURE OF THE SUBRIFT MAGMA CHAMBER}

The Southwest Indian Ridge is a slow-spreading axis $(0.8$ $\mathrm{cm} / \mathrm{yr}$ ) similar in many respects to the Mid-Atlantic Ridge. Nisbet and Fowler (1978) predicted that such slow-spreading ridges were underlain by small magma chambers, perhaps less than $2 \mathrm{~km}$ wide. Conversely, this model fits seismic evidence that strongly suggests the presence of large magma chambers, such as those found on the fast-spreading East Pacific Rise (Detrick et al., 1987). Generation of the Leg 118 gabbros in a relatively small magma chamber might explain many of their observed features: (1) their poor layering, which suggests small volumes of magma; (2) fractionation and limited mixing of several batches of magma with individual chemical characteristics; (3) possible thickening of oceanic layer 3 by magmatic intrusion of sills and dikes near the ridge axis; (4) more extensive mixing needed to explain the large spectrum of compositions of the silicate minerals and the locally abundant ilmenite, apatite, and zircon.

\section{CONCLUSIONS}

The mineralogy and phase chemistry of Leg 118 gabbros suggest that they were formed by fractional crystallization of tholeiitic magmas. The cumulates range from troctolites (Units V and VI), through olivine gabbros and gabbronorites to ilmenite-rich gabbros and gabbronorites. A wide range of mineral compositions was observed; olivine and pyroxene range from $\mathrm{Mg}$-rich to $\mathrm{Fe}$-rich, and plagioclase ranges from highly calcic to moderately sodic. At least some degree of magma mixing is required to explain the formation of Fe-rich and incompatible element-rich cumulates. Calculated parental melt compositions for the troctolites suggest that they were relatively fractionated liquids. Relatively low $\mathrm{CaO}$ / $\mathrm{Al}_{2} \mathrm{O}_{3}$ ratios for these melts suggest that clinopyroxene, olivine, and plagioclase were fractionated at low-to-moderate pressures. Poor layering, small volumes of magma, and possible intrusions suggest formation of the gabbros in a relatively small magma chamber, perhaps 1 to $2 \mathrm{~km}$ wide. We conclude that a small degree of magma mixing in such a chamber is physically possible to explain the mineralogical data. 


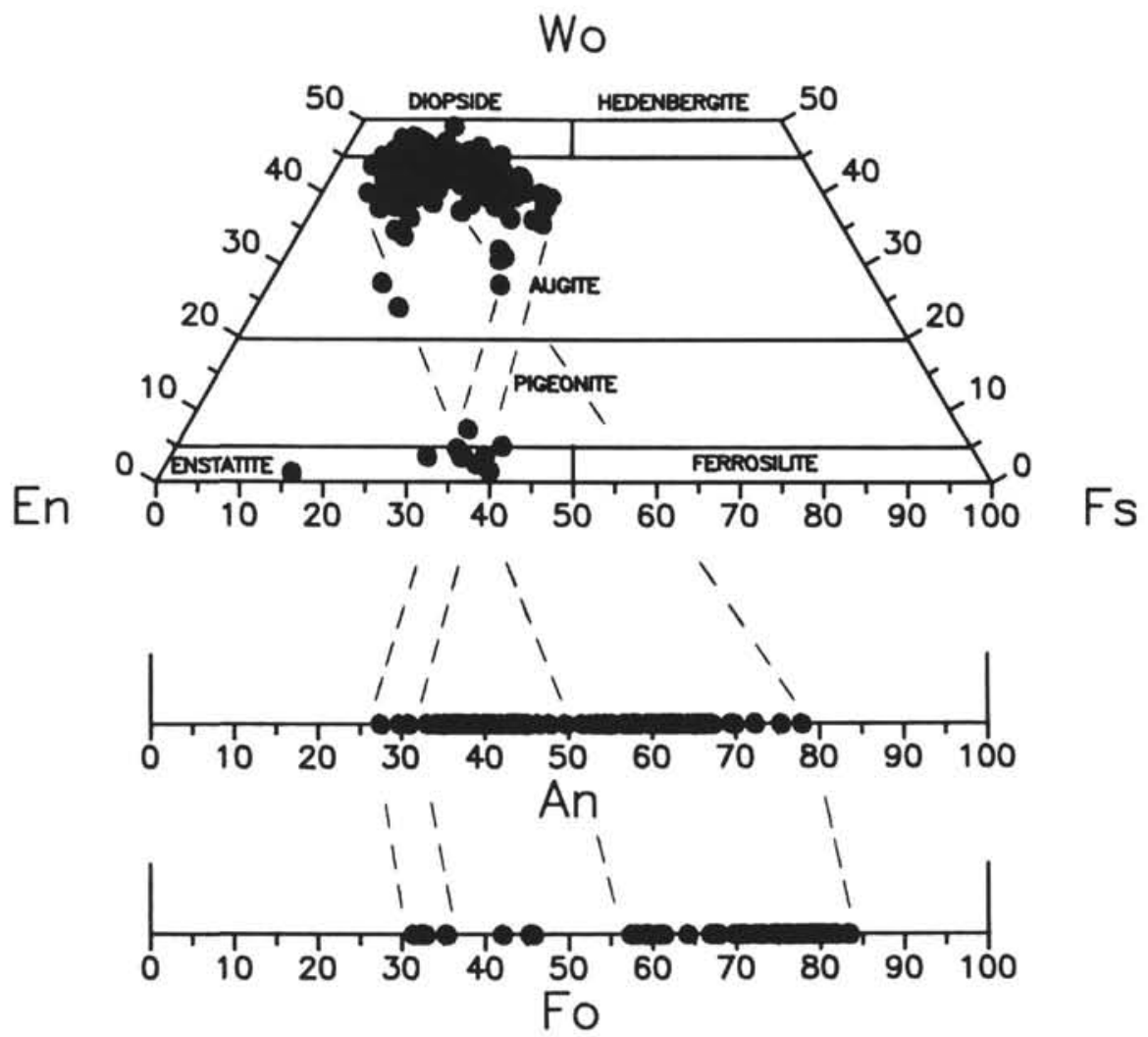

Figure 4. Compositional diagrams of clinopyroxene (Wo-En-Fs), plagioclase (An), and olivine (Fo). Scales are units of molecular proportions. Fields are defined after Subcommittee on Pyroxenes (1989).

\section{ACKNOWLEDGMENTS}

The authors (R. H., P.T.R.) benefitted from Natural Sciences and Engineering Research Council of Canada grants. We are indebted to all shipboard participants, and to D. K. Ross, and J. Longhi for their helpful comments and discussions. We acknowledge B. Mélançon for his computer-generated geochemical logs. We thank J. Pineau, who typed the manuscript.

\section{REFERENCES}

Bence, A. E., Baylys, D. M., Bender, J. F., and Grove, T. L., 1979. Controls on the major and minor element chemistry of mid-ocean ridge basalts and glasses. In Talwani, M., Harrison, C. G., and Hayes, D. E. (Eds.), Deep Drilling Results in the Atlantic Ocean: Ocean Crust: Washington (Am. Geophys. Union), Maurice Ewing Ser., 2:331-341.

Bryan, W. B., 1983. Systematics of modal phenocryst assemblages in submarine basalts: Petrologic implications. Contrib. Mineral. Petrol., 83:62-74.

Chalokwu, C. I., and Grant, N. K., 1987. Re-equilibration of olivine with trapped liquid in the Duluth complex, Minnesota. Geology, 15:71-74.

Colson, R. O., and Gust, D., 1989. Effects of pressure on partitioning of trace elements between low $\mathrm{Ca}$ pyroxene and melt. Am. Mineral., 74:31-36.

Detrick, R. S,, Bulh, P., Vera, E., Mutter, J., Orcutt, J. A., Madsen, J., and Brocher, T., 1987. Multichannel seismic imaging of an axial magma chamber along the East Pacific Rise between $9^{\circ} \mathrm{N}$ and $13^{\circ} \mathrm{N}$. Nature, $326: 35-47$.

Drake, M. J., 1976. Plagioclase-melt equilibrium. Geochim. Cosmochim. Acta, 40:457-465.

Eaby, J. D., Clague, D. A., and Eissen, J. P., 1986. Gabbroic xenoliths and host ferrobasalt from the Southern Juan de Fuca Ridge. J. Geophys. Res., 91:3795-3820.
Elthon, D., 1987. Petrology of gabbroic rocks from the Mid-Cayman Rise spreading center. J. Geophys. Res., 92:658-682.

Elthon, D., and Scarfe, C. M., 1984. High-pressure phase equilibria of a high-magnesia basalt and the genesis of primary oceanic basalts. Am. Mineral., 69:1-15.

Fujii, T., and Bougault, H., 1983. Melting relations of a magnesian abyssal tholeiite and the origin of MORBs. Earth Planet. Sci. Lett., 62:283-295.

Grove, T. L., and Bryan, W. B., 1983. Fractionation of pyroxenephyric MORB at low pressure: An experimental study. Contrib. Mineral. Petrol., 89:293-309.

Hébert, R., 1982. Petrography and mineralogy of oceanic peridotites and gabbros: Some comparisons with ophiolite examples. Ofioliti, 7:299-324.

1985. Pétrologie des roches ignées océaniques et comparaison avec les complexes opiolitiques du Québec, de Chypre et de l'Apennin [Ph.D. dissert]. Université de Bretagne Occidentale, Brest.

Hébert, R., Bideau, D., and Hekinian R., 1983. Ultramafic and mafic rocks from the Garret Transform Fault near $13^{\circ} 30^{\prime} \mathrm{S}$ on the East Pacific Rise: Igneous petrology. Earth Planet. Sci. Lett., 65:107-125.

Hébert, R., and Laurent, R., in press. Mineral chemistry of the plutonic section of the Troodos ophiolite: new constraints for genesis of arc-related ophiolites. Proc. Symp. Troodos '87, Geol. Surv. Cyprus (Nicosia).

Hébert, R., Serri, G., and Hekinian, R., 1989. Mineral chemistry of ultramafic tectonites and ultramafic to gabbroic cumulates from the major oceanic basin and the Northern Apennines Ophiolites-a comparison. Chem. Geol., 77:183-207.

Hekinian, R., Hébert, R., Maury, R. C., and Berger, E. T., 1985. Orthopyroxene-bearing gabbroic xenoliths in basalts from the East Pacific Rise axis near $12^{\circ} 50^{\prime}$ N. Bull. Minéral., 108:691-698.

Hekinian, R., Moore, J. G., and Bryan, W. B., 1976. Volcanic rocks and processes of the Mid-Atlantic Ridge rift valley near $36^{\circ} 49^{\prime} \mathrm{N}$. Contrib. Mineral. Petrol., 58:83-110. 
Hodges, F. N., and Papike, J. J., 1976. DSDP Site 334: Magmatic cumulates from oceanic layer 3. J. Geophys. Res., 81:41354151.

Irving, A. J., 1978. A review of experimental studies of crystal-liquid trace element partitioning. Geochim. Cosmochim. Acta, 42:743770 .

Komor, S. C., Elthon, D., and Casey, J. F., 1985. Mineralogy variation in a layered ultramafic cumulate sequence at the North Arm Mountain Massif, Bay of Islands Ophiolite, Newfoundland. J. Geophys. Res., 90:7705-7736.

, 1987. Petrology of a leuco-gabbroic interval in basal layered gabbros at North Arm Mountain, Bay of Islands ophiolite. Contrib. Mineral. Petrol., 95:278-300.

Lindslay, D. H., and Anderson, D. J., 1983. A two-pyroxene thermometer. Proc. 13th Lunar Planet. Sci. Conf., Part 2. J. Geophys. Res., 88A:887-906.

Longhi, J., 1982. Effects of fractional crystallization and cumulates processes on mineral composition trends of some lunar and terrestrial rock series. Proc. 13th Lunar Planet. Sci. Conf. Part 1. J. Geophys. Res., 87A:54-64.

McKenzie, D., and Bickle, M. T., 1988. The volume and composition of melt generated by extension of the lithosphere. J. Petrol., 23:625-670.

Mével, C., 1987. Evolution of oceanic gabbros from DSDP Leg 82: Influence of the fluid phase on metamorphic crystallizations. Earth Planet. Sci. Lett., 83:67-79.

Meyer, P. S., Ozawa, K., and Bloomer, S. H., 1989. Solidification of oceanic cumulates: Evidence from chemical maps of thin sections and chemical mass balances. EOS, 70:1400.

Morel, J. M., 1979. Evolution magmatique le long des dorsales Médio-Atlantique et Est-Pacifique. [M.S. thesis]. Université de Bretagne Occidentale, Brest.

Nicolas, A., Reuber, I., and Benn, K., 1988. A new magma chamber model based on structural studies in the Oman Ophiolite. Tectonophysics, 151:87-105.

Nisbet, E. G., and Fowler, C.M.R., 1978. The Mid-Atlantic Ridge at $37^{\circ}$ and $45^{\circ} \mathrm{N}$ : Some geophysical and petrological constraints. Geophys. J.R. Astron. Soc., 54:631-660.

Pallister, J. S., and Hopson, C. A., 1981. Samail ophiolite plutonic suite: Field relations, phase variation, cryptic variation and layering, and a Field relations phase variation, a model of a spreading ridge magma chamber. J. Geophys. Res., 86:2593-2644.
Pearce, J. A., and Norry, M. J., 1979. Petrogenetic implications of Ti, $\mathrm{Zr}, \mathrm{Y}$, and $\mathrm{Nb}$ variations in volcanic rocks. Contrib. Mineral. Petrol., 69:33-47.

Presnall, D. C., Dixon, S. A., Dixon, J. R., O'Donnell, T. H., Brenner, N., Schrock, R. L., and Dycus, D. W., 1978. Liquidus phase relations to the joint diopside-forsterite-anorthite from $1 \mathrm{~atm}$ to 20 kbar: their bearing on the generation and crystallization of basaltic magmas. Contrib. Mineral. Petrol., 66:203-220.

Reid, J. B., Steig, E., and Bryan, W. B., 1989. Major element evolution of basaltic magmas: A composition of the information in CMAS and ALFE projections. Contrib. Mineral. Petrol., 101:318325.

Robinson, P., 1980. The composition space of terrestrial pyroxenes. Internal and external limits. In Prewitt, C. T. (Ed.), Pyroxenes. Min. Soc. Am. Rev. Mineral., 7:419-475.

Robinson, P. T., Von Herzen, R. P., et al. 1989. Proc. ODP, Init. Repts., 118: College Station, TX U.S.A. (Ocean Drilling Program).

Roeder, R., and Emslie, F., 1970. Olivine-liquid equilibrium. Contrib. Mineral. Petrol., 29:275-289.

Sato, H., 1977. Nickel content of basaltic magmas: Identification of primary magmas and a measure of the degree of olivine fractionation. Lithos, 10:113-120.

Stakes, D. S., Shervais, J. W., and Hopson, C. A., 1984. The volcanic-tectonic cycles of the FAMOUS and AMAR valleys, MidAtlantic Ridge $\left(36^{\circ} 47^{\prime} \mathrm{N}\right)$ : evidence from basalt glass and phenocryst compositional variations for a steady-state magma chamber beneath the valley midsections, AMAR 3. J. Geophys. Res., 89:6995-7028.

Subcommittee on Pyroxenes, 1989. Nomenclature of pyroxenes. Can. Mineral., 27:143-156.

Ulmer, P., 1989. The dependence of the $\mathrm{Fe}^{2+}-\mathrm{Mg}$ cation-partitioning between olivine and basaltic liquid on pressure, temperature and composition: an experimental study to $30 \mathrm{kbars}$. Contrib. Mineral. Petrol., 101:261-273.

Date of initial receipt: 11 July 1989

Date of acceptance: 21 February 1990

Ms 118B-119 

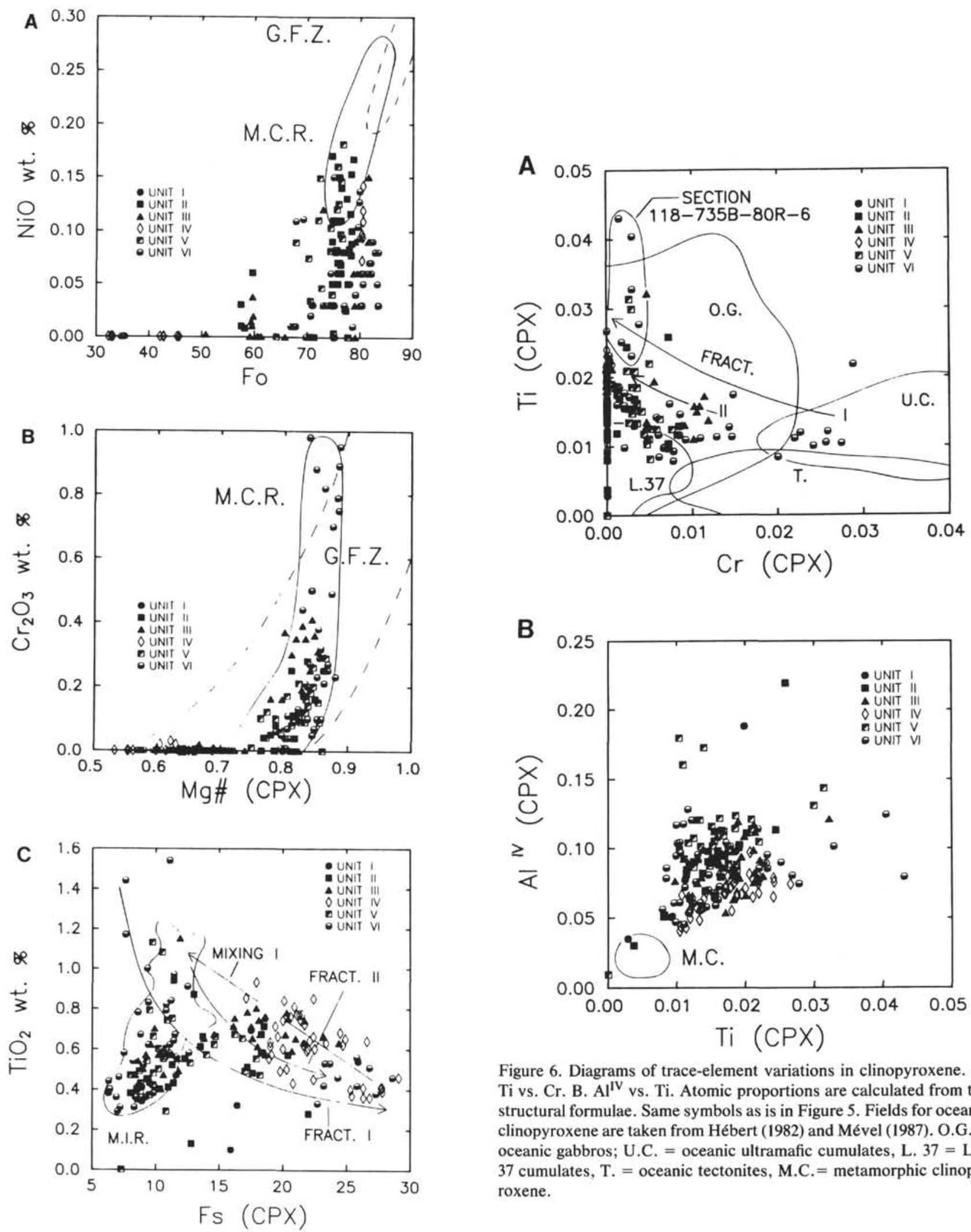

Figure 6. Diagrams of trace-element variations in clinopyroxene. A. Ti vs. Cr. B. Al ${ }^{\mathrm{IV}}$ vs. Ti. Atomic proportions are calculated from the structural formulae. Same symbols as is in Figure 5. Fields for oceanic clinopyroxene are taken from Hébert (1982) and Mével (1987). O.G. = oceanic gabbros; U.C. = oceanic ultramafic cumulates, L. $37=\mathrm{Leg}$ 37 cumulates, $\mathrm{T} .=$ oceanic tectonites, M.C. $=$ metamorphic clinopyroxene.

Figure 5. Diagrams of element contents vs. mineral molecular proportions. A. $\mathrm{NiO}$ wt $\%$ vs. Fo content of olivine. $\mathrm{B} . \mathrm{Cr}_{2} \mathrm{O}_{3}$ wt $\%$ vs. $\mathrm{Mg} \#$ in clinopyroxene. $\mathrm{C}$. $\mathrm{TiO}_{2}(\mathrm{wt} \%)$ vs. Fs content in clinopyroxene. Symbols correspond to the six lithologic units. M.I.R. = MidIndian Ridge gabbroic rocks. Fields from Elthon (1987) and Hébert et al. (1983). For explanations see text. 

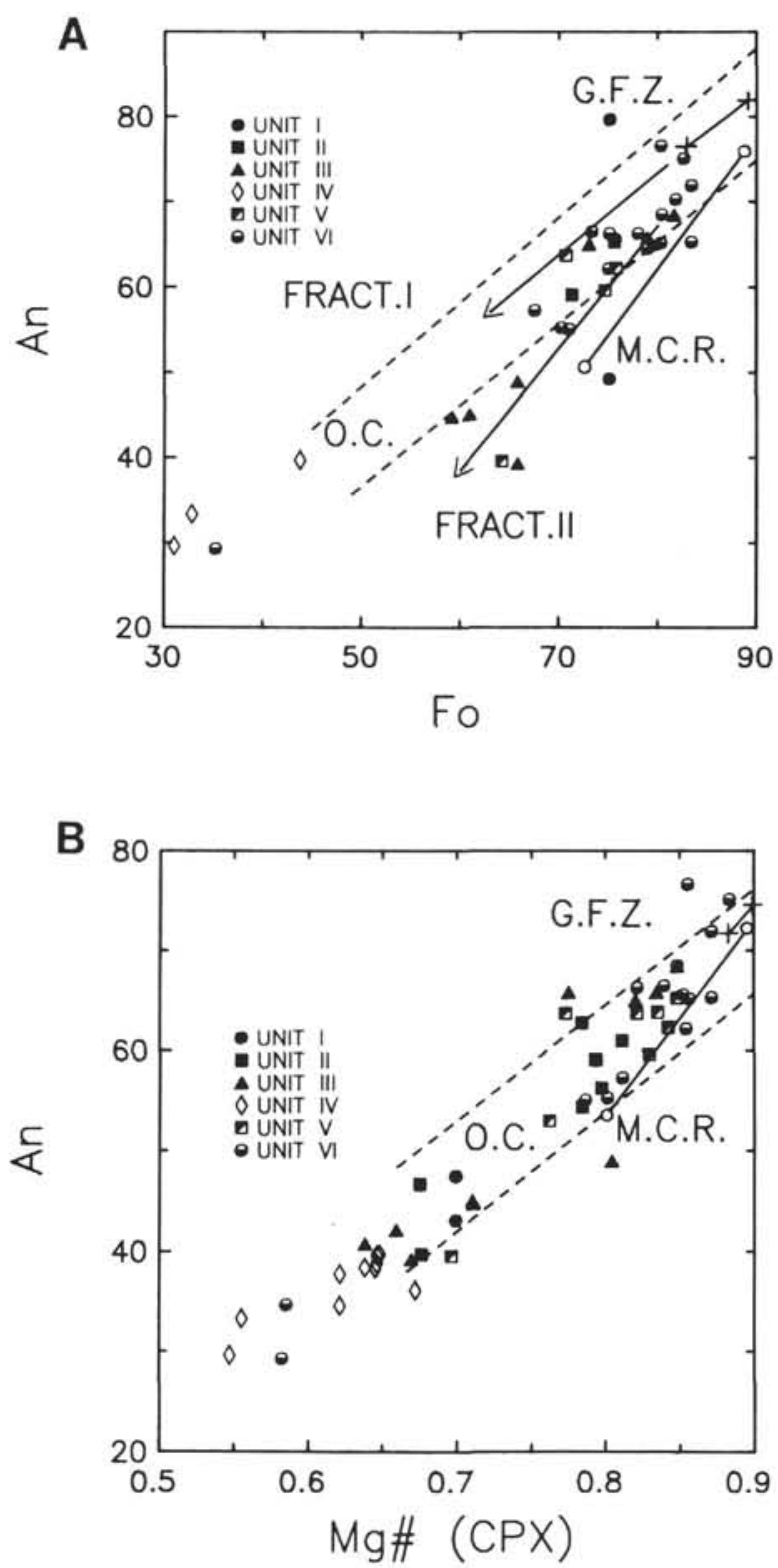

Figure 7. Diagrams of mineral co-variation. A. Anorthite content in plagioclase vs. Fo content in olivine. B. Anorthite content in plagioclase vs. $\mathrm{Mg} \#$ in clinopyroxene. Trend of evolution for GFZ is taken from Hébert et al. (1983). Trend evolutions for MCR are taken from Elthon (1987). Field of oceanic spectrum is taken from Hébert et al. (1989). O.C. $=$ oceanic cumulates. Other symbols as in Figures 5 and 6. 


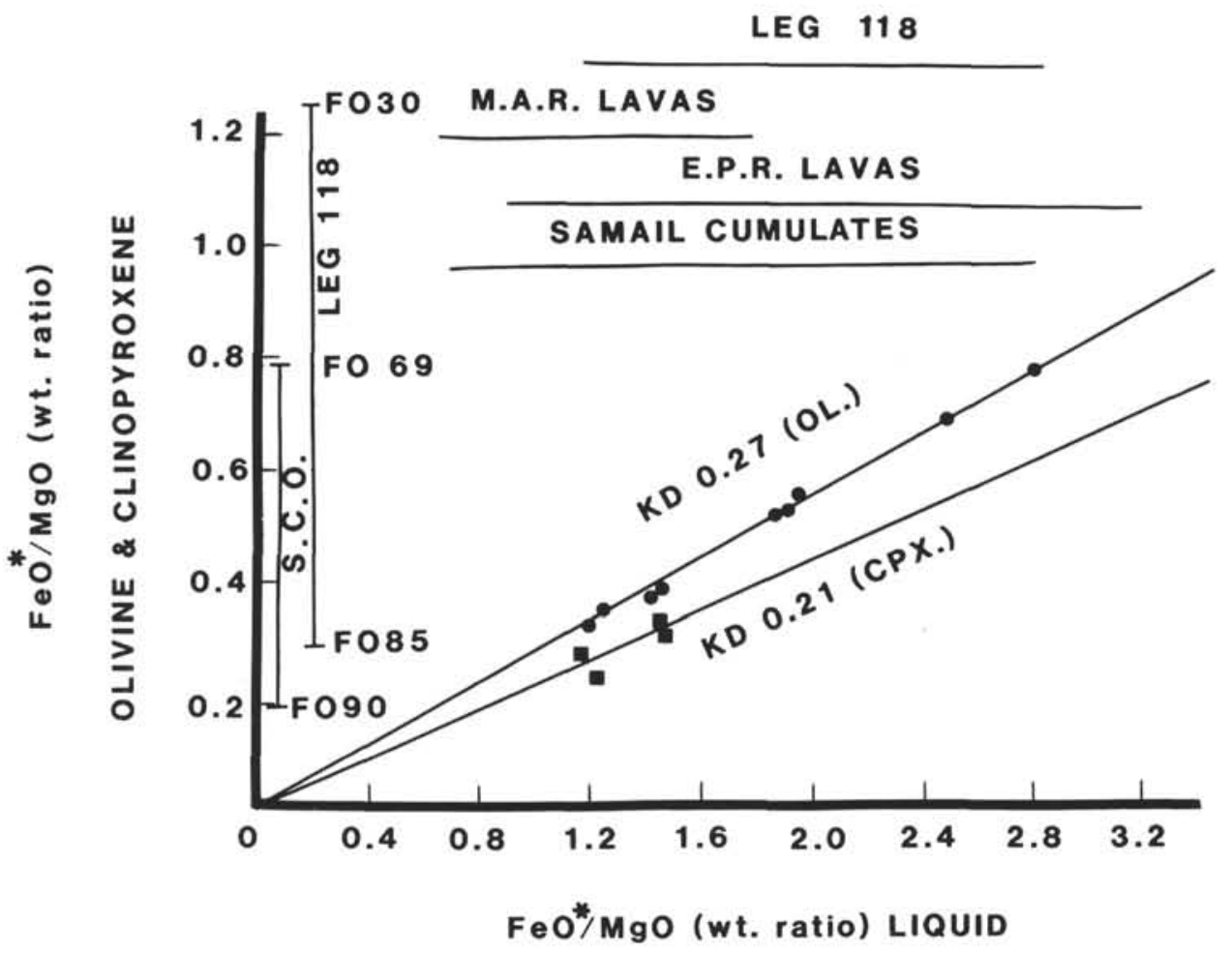

Figure 8. Diagram of $\mathrm{FeO}^{*} / \mathrm{MgO}$ ratio in olivine and clinopyroxene vs. $\mathrm{FeO} * / \mathrm{MgO}$ wt. ratio in coexisting liquid. Ranges of variation for Pacific MOR lavas and Oman ophiolite are reproduced from Pallister and Hopson (1981). SCO = Samail cumulates ophiolite. MAR = Mid-Atlantic Ridge, EPR $=$ East Pacific Rise. 


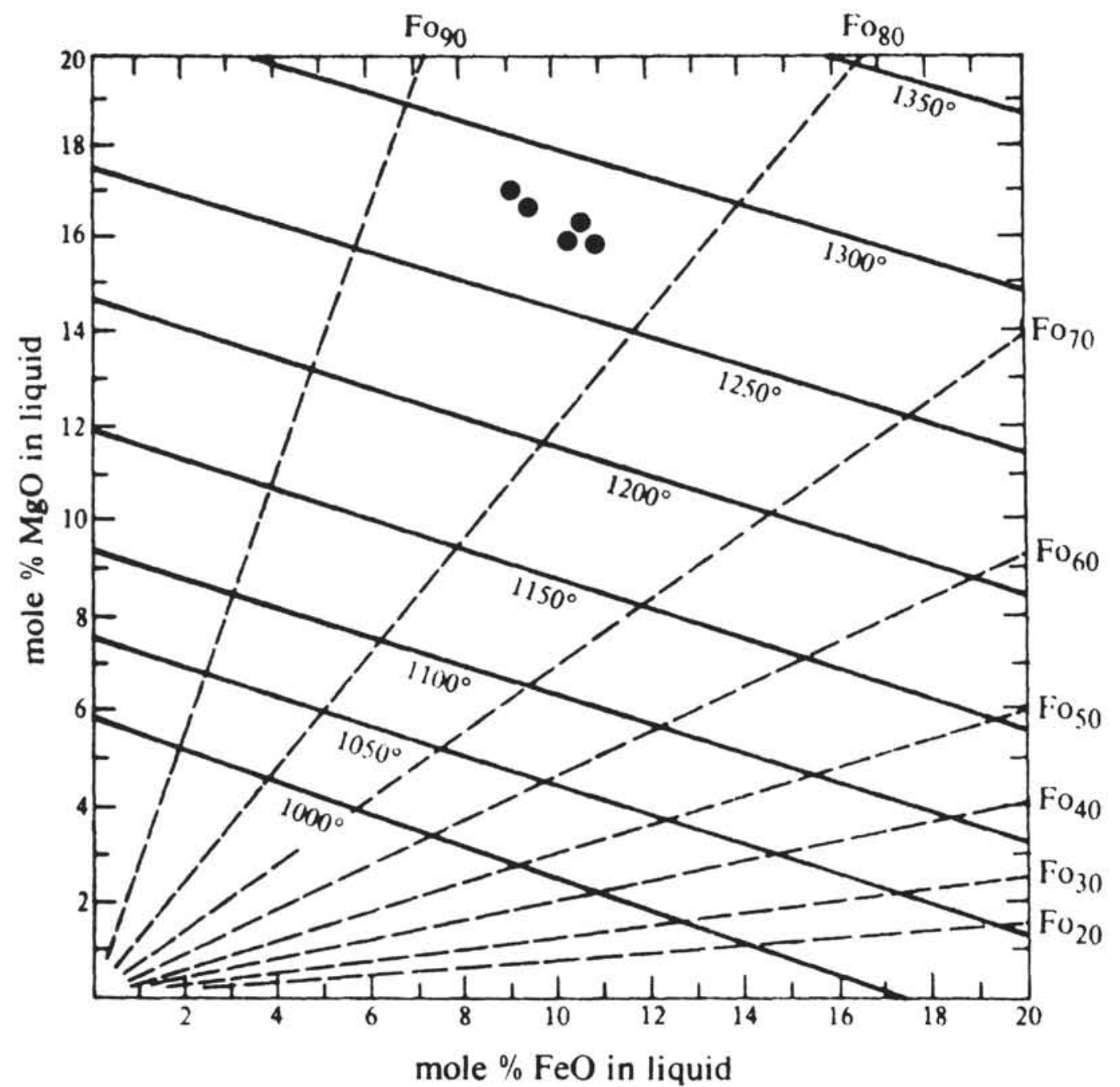

Figure 9. Diagram of molar percentage of $\mathrm{MgO}$ vs. molar percentage of $\mathrm{FeO}$ in calculated liquids in equilibrium with cumulus olivine from Unit VI (after Roeder and Emslie, 1970). 